Swarthmore College

Works

1996

\title{
Visual Learning In The Perception Of Texture: Simple And Contingent Aftereffects Of Texture Density
}

Frank H. Durgin

Swarthmore College, fdurgin1@swarthmore.edu

D. R. Proffitt

Follow this and additional works at: https://works.swarthmore.edu/fac-psychology

Part of the Psychology Commons

Let us know how access to these works benefits you

\section{Recommended Citation}

Frank H. Durgin and D. R. Proffitt. (1996). "Visual Learning In The Perception Of Texture: Simple And Contingent Aftereffects Of Texture Density". Spatial Vision. Volume 9, Issue 4. 423-474. DOI: 10.1163/ $156856896 \times 00204$

https://works.swarthmore.edu/fac-psychology/134

This work is brought to you for free by Swarthmore College Libraries' Works. It has been accepted for inclusion in Psychology Faculty Works by an authorized administrator of Works. For more information, please contact myworks@swarthmore.edu. 


\section{Texture Density Adaptation}

Draft version

Published version: Spatial Vision (1996), 9, 423-474

\section{Visual Learning in the Perception of Texture:}

\section{Simple and Contingent Aftereffects of Texture Density}

\section{Frank H. Durgin* and Dennis R. Proffitt**}

* Department of Psychology, Swarthmore College, Swarthmore, PA 19081, USA

** Department of Psychology, University of Virginia, Charlottesville, VA 22903, US

Send correspondence and preprint requests:fdurgin1@swarthmore.edu

Frank H. Durgin

Department of Psychology

Swarthmore College

500 College Avenue

Swarthmore, PA 19081

PHONE: (610) 328-8678

FAX: (610) 328-7814

\section{Abstract}

Novel results elucidating the magnitude, binocularity and retinotopicity of aftereffects of visual texture density adaptation are reported as is a new contingent aftereffect of texture density which suggests that the perception of visual texture density is quite malleable. Texture aftereffects contingent upon orientation, color and temporal sequence are discussed. A fourth effect is demonstrated in which auditory contingencies are shown to produce a different kind of visual distortion. The merits and limitations of error-correction and classical conditioning theories of contingent adaptation are reviewed. It is argued that a third kind of theory which emphasizes coding efficiency and informational considerations merits close attention. It is proposed that malleability in the registration of texture information can be understood as part of the functional adaptability of perception. 
In order to maintain its wonderful illusion of direct and complete perception, the visual system must find ways to efficiently and accurately encode information. This problem seems of particular importance in the perception of visual details, such as textures, which seem to exist in such astonishing variety that their representation appears to have endless dimensionality. Casual introspection suggests that we seem to perceive textures in full detail, but this 'seeming' is suspicious. That this seemingly direct perception of texture is an illusion is made starkly evident by the aftereffects of texture density to be described in this paper. Still, our ability to identify and discriminate visual textures is impressive. What processes does the perceptual representation of texture depend on to be as successful as it normally is?

One might expect perceptual learning to play an important role in the perception of visual texture because the visual system must choose how to represent texture. It ought to do it wisely and therefore adaptively. Indeed, evidence of perceptual learning has been reported, both in the learned improvement of texture discrimination (Karni and Sagi, 1991, 1993) and in the choice of coding mechanisms (e.g. Field, 1994; Foldiak, 1990) for the representation of textures. The purpose of the present article is to describe another kind of evidence of perceptual learning in the perception of texture that has emerged from work performed in our laboratories on the adaptation of perceived texture density.

To accomplish our goal, our paper is divided into three distinct parts: To set the stage for understanding the contingent adaptation of texture perception, we will first provide a description of simple texture density adaptation and present data demonstrating some properties of the density aftereffect in the non-Fourier domain. Although there have been previous reports of texture density aftereffects (e.g. Anstis, 1974; Walker, 1966), none have heretofore distinguished density from spatial frequency (i.e. texture size).

In the second portion of the paper we will review two contingent aftereffects of texture density recently reported by our laboratories and report a new one. To preview, Durgin (in press) has shown that perceived texture density can be made contingent on the color of a surrounding region, and Durgin and Hammer $(1994,1995)$ have made both texture density and texture brightness contingent on the temporal order of texture presentation. We will also describe experiments in which we have investigated the extent to which texture density can be made contingent on texture element orientation, and, in addition to these contingent aftereffects, we will report the results of an experiment in which adaptation to intermodal contingencies produced a positive (assimilatory) aftereffect.

The reason we will focus on contingent adaptation is that several models of efficient perceptual processing make appeals to analyses of environmental contingencies (e.g. Barlow and Foldiak, 1989; Helson, 1964). It has been our goal to artificially manipulate various environmental contingencies and to examine the effects of these manipulations on the perceptual experience of visual texture. We will argue that the visual perception of texture draws upon experience in a variety of ways that are both surprising and are perhaps achieved very simply.

In the final section of the article, we will evaluate several theoretical models of contingent adaptation and compare contingent adaptation to other forms of visual learning. Having elucidated the nature of density aftereffects and demonstrated a variety of contingent density aftereffects, we will then try to demonstrate that there are common principles of efficient information representation pertaining to the representation of visual texture and to its adaptation.

\section{The Perception of Texture Density: Studies in Adaptation.}

It has been recognized for some time that spatial-frequency (Fourier) analysis captures important aspects of the human visual system's analysis of texture. However, one non-Fourier spatial property of textures, element density, has been recently shown, by its adaptation, to also be implicated in the perceptual representation of texture (Durgin and Proffitt, 1991; Durgin, 1995a, 1995b). Prior demonstrations of texture density adaptation had been based on texture magnification (e.g. MacKay, 1964; Walker, 1966) and attributed to spatial-frequency channel adaptation (Anstis, 1974), but the same effects can be observed with stimuli in which both luminance and spatial frequency are 
kept constant.

In Fig. 1 is shown the earlier version of the texture density aftereffect which can be understood as a spatialfrequency aftereffect in the perception of texture (Anstis, 1974). Adaptation to the pair of textures in the left panel (while gazing at the central fixation mark for several seconds) will produce a negative aftereffect which can be experienced by rapidly switching to the right panel; because the upper region is denser in the left panel, the upper region will probably seem less dense than the lower region. By magnifying textures, average luminance was held constant but element size was varied.
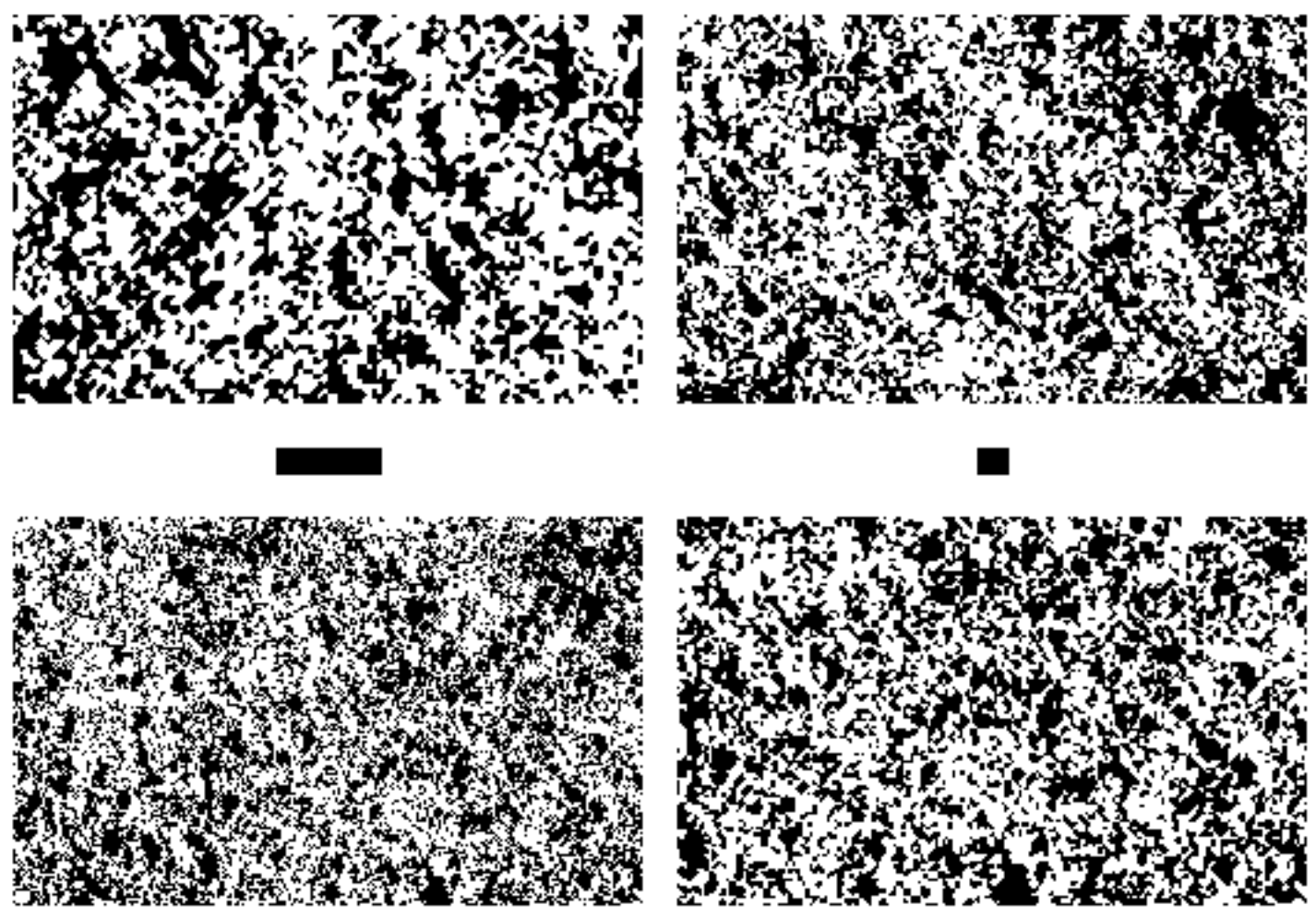

Figure 1. Demonstration of texture size/density aftereffect (after Anstis, 1974). To experience the aftereffect, gaze at the left fixation mark for several seconds (20-60), moving eyes along fixation bar to avoid the formation of afterimages. Then quickly shift gaze to right fixation square and compare the apparent density of the two right-hand textures. The lower texture should now appear less dense (coarser) than the upper texture. Note that in this demonstration, density is manipulated by texture magnification and, as a result, is confounded with size. Anstis (1974) argued that density aftereffects were due, in part, to spatial frequency adaptation. 


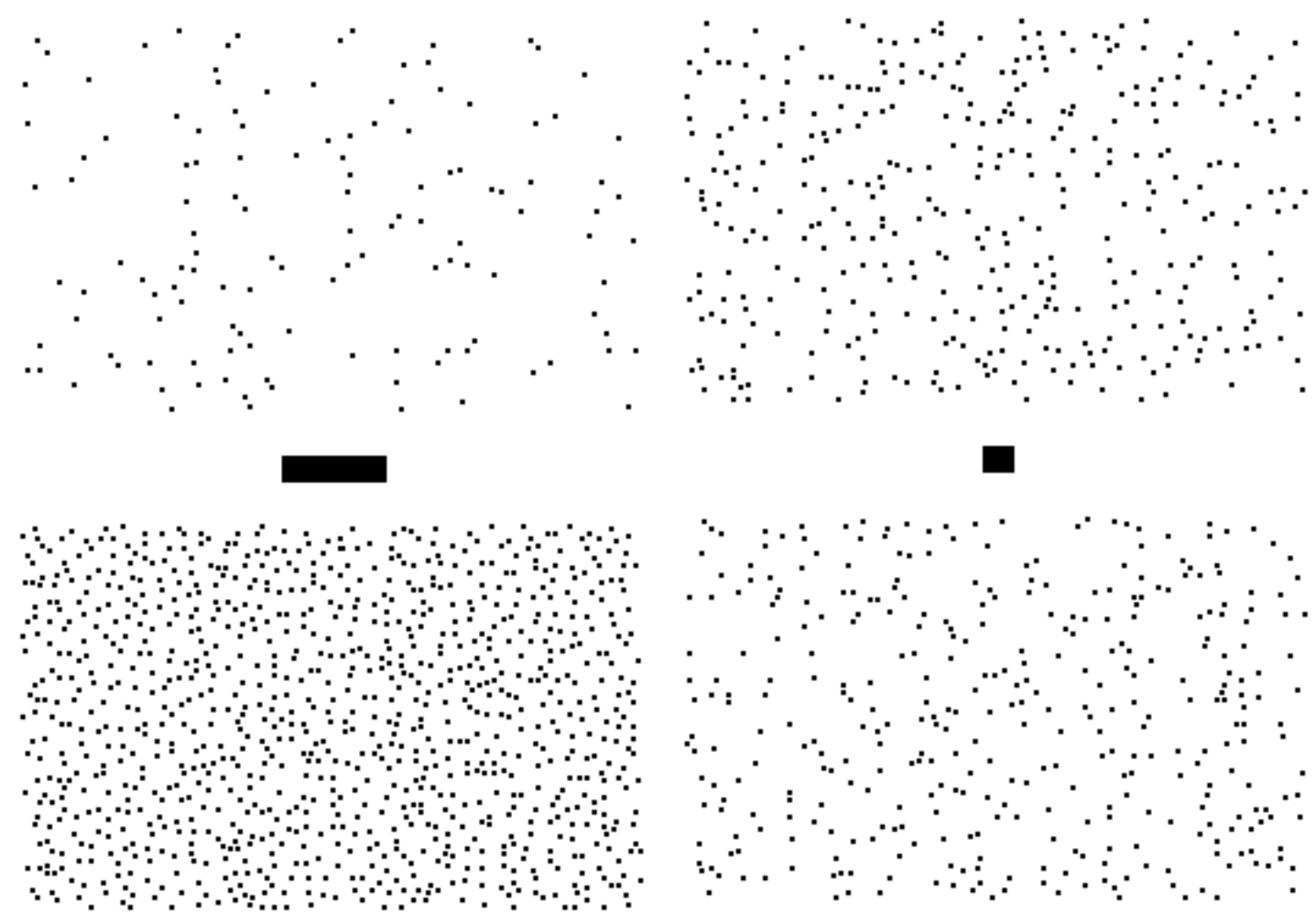

Figure 2. Demonstration of texture density aftereffect without size confound (after Durgin and Proffitt, 1991). To experience aftereffect, follow procedure as in Fig. 1. After adapting to the left pair, the upper texture on the right should appear denser than the lower one. Note that in this demonstration, density is not confounded with element size or spatial frequency because element size is held constant. Although density is confounded with luminance, this can be remedied by the use of luminance-balanced texture elements. (See Fig. 3).

Figure 2 illustrates a related aftereffect in which density is varied without varying element size (Durgin and Proffitt, 1991). Following the same procedure as for Fig. 1, an aftereffect of apparent texture density can be observed in this condition as well, though the Fourier transforms of the two adaptation fields have similar spectral properties. In this case there are obviously space-average luminance differences between the two fields, but these are probably of no consequence. Although Mulligan and MacLeod (1988) and Cornelissen and Kooijman (1994) have suggested that density and brightness modulations (with unbalanced dots) may in part be interchangeable (cf., also Chubb, Sperling, and Solomon, 1989), Durgin (1995a; Durgin and Hammer, 1994) has found that texture brightness aftereffects (from contrast adaptation) and texture density aftereffects show little inter-dimension transfer and have different patterns of interocular transfer (cf. also Burgess and Barlow, 1983). 


\begin{tabular}{|c|c|c|c|c|c|c|c|}
\hline $1 \mathrm{~L}$ & $1 \mathrm{~L}$ & $1 \mathrm{~L}$ & $1 \mathrm{~L}$ & $1 \mathrm{~L}$ & $1 \mathrm{~L}$ & $1 \mathrm{~L}$ & \multirow{7}{*}{ 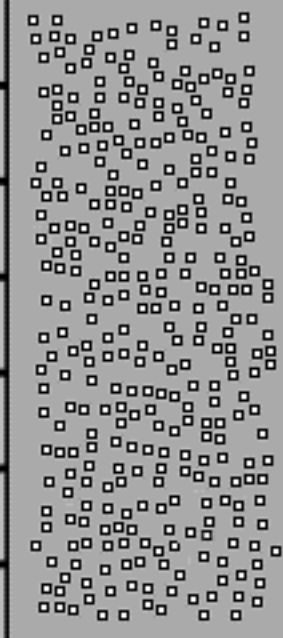 } \\
\hline $1 \mathrm{~L}$ & $\mathrm{OL}$ & OL & OL & $\mathrm{OL}$ & $1 \mathrm{~L}$ & $1 \mathrm{~L}$ & \\
\hline $1 \mathrm{~L}$ & $\mathrm{OL}$ & $4 \mathrm{~L}$ & $4 \mathrm{~L}$ & $\mathrm{OL}$ & $1 \mathrm{~L}$ & $1 \mathrm{~L}$ & \\
\hline $1 \mathrm{~L}$ & $\mathrm{OL}$ & $4 \mathrm{~L}$ & $4 \mathrm{~L}$ & $O L$ & $1 \mathrm{~L}$ & $1 \mathrm{~L}$ & \\
\hline $1 \mathrm{~L}$ & $\mathrm{OL}$ & $\mathrm{OL}$ & OL & $\mathrm{OL}$ & $1 \mathrm{~L}$ & $1 \mathrm{~L}$ & \\
\hline $1 \mathrm{~L}$ & $1 \mathrm{~L}$ & $1 \mathrm{~L}$ & $1 \mathrm{~L}$ & $1 \mathrm{~L}$ & $1 \mathrm{~L}$ & $1 \mathrm{~L}$ & \\
\hline $1 \mathrm{~L}$ & $1 \mathrm{~L}$ & $1 \mathrm{~L}$ & $1 \mathrm{~L}$ & $1 \mathrm{~L}$ & $1 \mathrm{~L}$ & $1 \mathrm{~L}$ & \\
\hline
\end{tabular}

Figure 3. Schematic illustration of pixel structure of a balanced dot (left) and a representation of a dense balanced dot texture (right). In left image, theoretical luminance values for individual pixels are meant to illustrate the fact that the space-average luminance of the dot $(L)$ is maintained in the dot, though it is a high-contrast stimulus. A small patch of dense texture is shown at right (337 dots are shown).

In order to demonstrate that the effect in Fig. 2 could be obtained even when luminance differences were not present, we have created dot-textures composed of balanced-dot elements. Figure 3 depicts both the pixel structure of a single square balanced dot and a representative illustration of a balanced dot texture. Round balanced dots were originally used by Carlson, Moeller and Anderson (1984) to investigate geometrical illusions of size in the absence of low-spatial frequencies. Although non-linearities in visual processing (such as logarithmic transformations of input luminances at the retina) can render ostensibly balanced dots unbalanced (cf. Garcia-Perez, 1991), they represent a fairly well-filtered stimulus, and have been used effectively in investigations of motion processing (Gilden, Bertenthal and Othman, 1990).

\section{Overview of simple density aftereffect experiments.}

The three experiments to be reported concerning simple texture density adaptation are previously unpublished(Note 1) demonstrations that the texture density aftereffect is obtainable with balanced-dot textures. The first experiment we report here assesses the magnitude of the aftereffect both when observers were readapted immediately before each measurement trial and, following this, when they were retested without further readaptation. The magnitude of the effect, which will be shown to approximate a proportional reduction in perceived density, is striking in both conditions.

In the second experiment the contribution of peripheral factors to the short-term density aftereffect is investigated by dichoptic presentation of the adaptation and testing stimuli. The effects of very short-term adaptation do not show complete interocular transfer, though previous evidence (Durgin, 1992) suggests that the longer-term aftereffects do.

In a third experiment the retinotopicity of the effect is investigated. In order to test a classical conditioning hypothesis of the effect we dissociated screen position and retinal position in order to show that retinal, not screen, position determined the presence of the aftereffect.

Because the same basic methodology for inducing and testing visual aftereffects of texture density will be used in all of the studies in this section and others that follow, an outline of our general method is provided here. 


\section{General Method}

The subjects for the experiments reported here were primarily students at the University of Virginia or at Swarthmore College. Most experiments were conducted using Sun Microsystems computer workstations and color monitors with $40 \mathrm{pixel} / \mathrm{cm}$ resolution.

\section{Stimuli}

Unless otherwise specified, the textures used were composed of randomly scattered square balanced dots. Each dot consisted of a central 2- by 2-pixel square, usually white, surrounded by a 1-pixel wide, usually black, annulus. The dots were scattered over a homogenous gray background with the same space-average luminance as the dots themselves as determined by photometric measurement(Note2). The dots were constrained not to touch or overlap with one another, so that the resulting texture was composed of discrete elements as depicted in the lower portion of Fig. 3. Each presented texture was always a newly generated random texture. Stimulus duration was $1000 \mathrm{~ms}$ unless otherwise indicated, with a 1000-ms ISI during adaptation and re-adaptation.

\section{Adaptation}

For most experimental conditions, adaptation was performed under conditions of binocular viewing with a central fixation target. On each adaptation trial, two textures were presented in locations that were usually symmetrical about the fixation mark. One of the textured region was consistently quite dense and the other sparse. The presence of the sparse texture, which does not by itself produce any significant visual distortion (Durgin and Proffitt, 1991), was primarily to maintain some texture processing (and equivalent contrast processing) in both regions to be tested. Typically, several hundred adaptation trials were given initially. Often, two or three re-adaptation trials were presented prior to each measurement trial to keep the aftereffect saturated. Our method of many repeated adaptation trials was inspired by Wolfe's (1990) studies of tilt aftereffects.
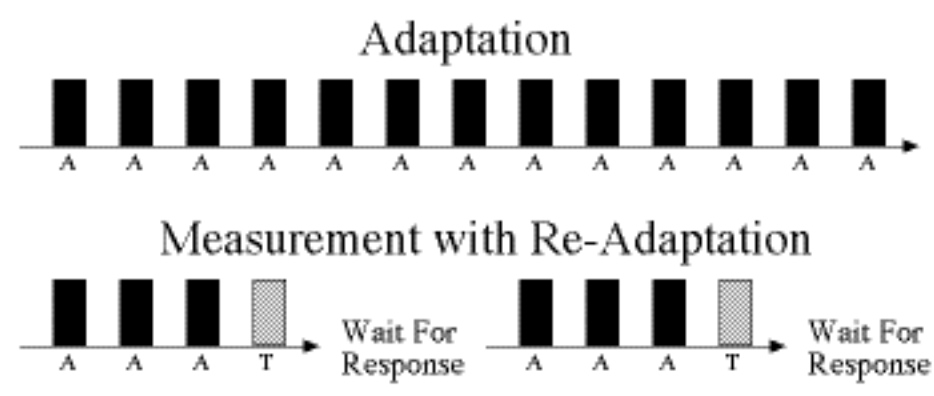

Measurement without Re-Adaptation

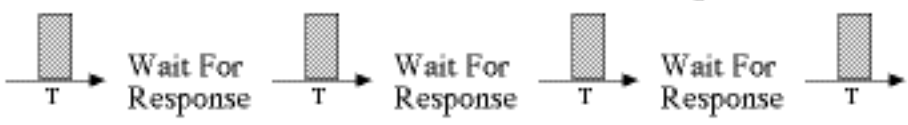

Figure 4: General time course of adaptation and measurement trials. Exact temporal parameters vary from one experiment to another, but typical stimulus presentation time was $1 \mathrm{~s}$ exposure with a $1 \mathrm{~s}$ ISI during adaptation. Duration of adaptation stimuli $(A)$ is depicted by black rectangles, duration of test stimuli $(T)$ by hatched rectangles. 


\section{Measurement}

The observer's task on each measurement trial was to choose which of two presented textures appeared denser. Points of subjective equality (PSE) for perceived density were measured for each subject by means of a modified staircase (up-down) procedure. The observer's responses determined the next stimulus associated with that staircase in the following way: the density of one of the two fields was adjusted by some pre-determined amount (a 'step') in a direction that will offset the reported difference between the textures. In this way, observers's judgments will tend to produce the presentation of textures which are subjectively similar in density. A 'turn' in a staircase consists of consecutive trials within that staircase to which the observer gives opposite responses (which results in a decrement followed by an increment or vice-versa).

Several PSEs were simultaneously calculated by interleaved staircases. To roughly synchronize the termination of the several staircases, the random selection of each staircase was weighted by the square of the number of turns remaining in the staircase. All staircases began with the presentation of textures that were objectively equal in density. Because effect sizes are typically quite large, the initial step size of the staircase procedures was $15 \%$ of the standard (unchanging) density. It was decreased to $10 \%$ after the first turn and then to a terminal value of $5 \%$ after the second turn in each staircase. Staircases were typically terminated after 8 turns and the PSE calculated as the mean number of dots in the comparison field at the last six turns of the staircase.

\section{Analysis}

Aftereffect size may be expressed as a ratio between the densities of textures presented in the two regions at the PSE, or as the logarithm of that ratio. Because the dense-adapted region appears less dense than the sparseadapted region, more dots are present in it at the PSE. Therefore, an aftereffect will be evidenced by a ratio between the dots in the dense- and sparse-adapted fields that is greater than 1. Statistical analyses are performed on the logarithm of these ratios because logarithms of ratios retain arithmetic properties appropriate for such analyses: The logarithm of a ratio and the logarithm of the reciprocal of that ratio are identical in absolute value, making the statistical analyses independent of the direction in which the ratios are computed. See Durgin, in press, for a detailed justification. The point of objective equality is a ratio of 1.0 which has a natural $\log$ of 0 . All data are graphed in logarithmic space, but aftereffect values are reported as (logarithms of) ratios in the text to facilitate appreciation of their magnitude.

\section{Experiment 1: Measuring the simple density aftereffect}

Purpose: The purpose of this experiment was to establish both that the density aftereffect could be obtained with balanced-dot textures and that the magnitude of the density aftereffect, expressed as a ratio, is constant across a broad range of densities (cf., Durgin, 1995b). In addition, we sought to compare the magnitude of the density aftereffect as measured with readaptation before each measurement trial and the magnitude when measured immediately thereafter without readaptation before each measurement trial.

\section{Method}

Eight graduate students and faculty at the University of Virginia (including the second author) served as observers. All eight were initially adapted, for four minutes, to texture fields which were dense above the fixation mark (1500 dots scattered over a region $\left.9^{\circ} \times 13^{\circ}\right)$ and sparse below it $\left(300\right.$ dots scattered over a region $\left.9^{\circ} \times 13^{\circ}\right)$. Their PSEs were then measured (with 3 readapting stimuli before each measurement trial) for four different densities: 200 or 900 
dots presented in the lower (less adapted) region, and 700 or 900 dots presented in the upper (dense-adapted) region. Five of these observers were then re-tested (within three to five minutes of completing the first session) for PSEs for the same densities, but without any further readaptation trials.

In addition, three University of Virginia undergraduates served as unadapted controls.

\section{Results and Discussion}

To determine whether aftereffect size varied as a function of density, separate repeated-measure ANOVAs were conducted for each condition (readaptation and no-readaptation) on the log(ratio) aftereffects scores at each density tested both for the scores with readaptation and without. In both cases, the log(ratio) aftereffect did not vary

systematically with the density of the fields tested; with readaptation: $F(3,7)=0.615$, ns, without readaptation: $F(3,4)$ $=0.453, \mathrm{~ns}$. The data for the eight observers who completed the test trials with readaptation are shown in Fig. 5 .

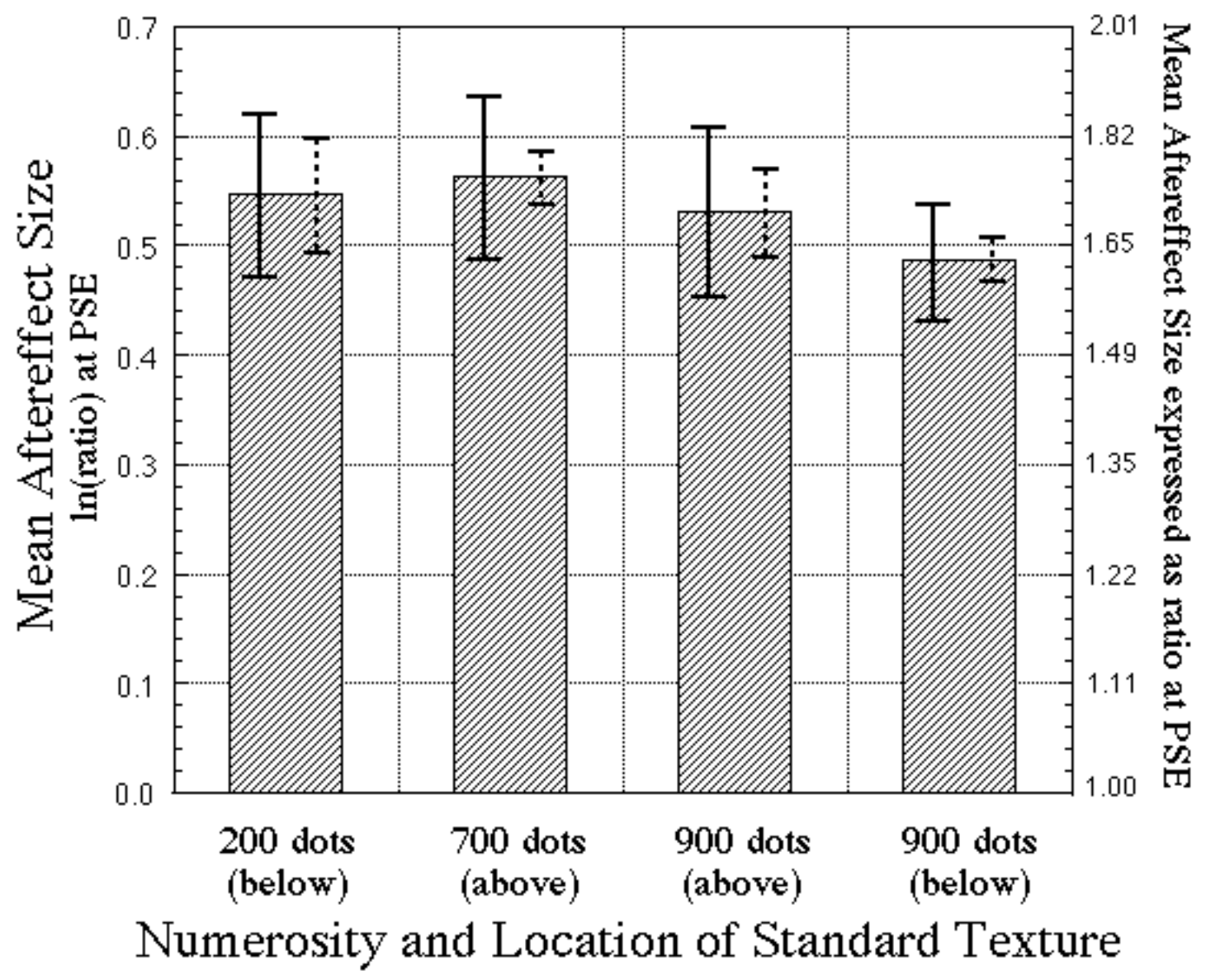

Figure 5: Results of readaptation condition of Experiment $1(\mathrm{~N}=8)$. Aftereffect size is represented by the mean of the natural logarithm of the ratio between the density of the upper and lower test fields at the point of subjective equality. Untransformed ratio values (number of dots in dense-adapted region divided by the number of dots in the other region at the point of subjective equality) are shown on the right axis. Solid error bars represent the between-subject standard error of the mean. Dashed error bars represent standard error with respect to within-subject effects. This was calculated by computing standard errors after removing overall between-subject differences in aftereffect size, and it provides a more appropriate basis for contrasting the various means. 
mean aftereffect score was reliably higher in the readaptation condition, $M=\log (1.66)$, than in the no-readaptation condition, $M=\log (1.32), F(1,4)=16.4, p<0.05$. Both conditions showed a marked bias towards seeing the spatially lower field as denser. These data are shown in Fig. 6. Although no baseline data were collected on these observers, three observers tested without adaptation revealed no reliable bias, with a mean PSE log(ratio) score of $\log (0.99)$.

Figure 6: Comparison of mean aftereffect sizes in Experiment 1 for sessions with readaptation preceding each measurement trial and for sessions without readaptation $(\mathrm{N}=5)$. Solid error bars represent the between-subject standard error of the mean. Dashed error bars represent standard error with respect to within-subject effects. (See caption of Fig. 4.)

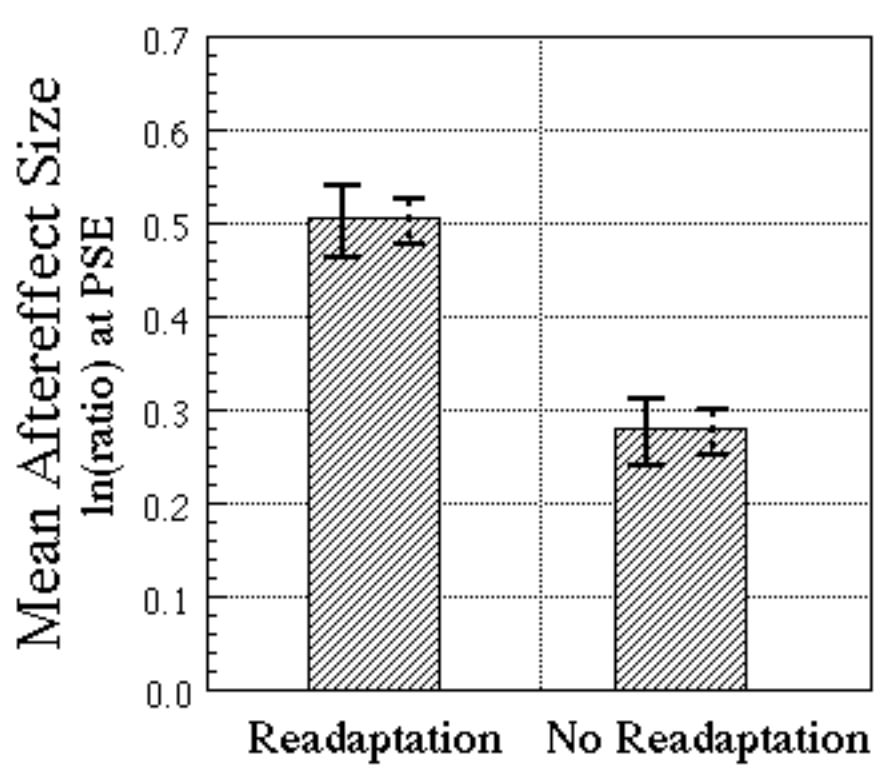

It is also possible to analyze whether the magnitude of the aftereffect changed during the time of testing by looking at whether the PSEs estimated by the earliest turns in the staircases differ from those at the final turns. Measurement lasted about ten minutes when conducted with readaptation and only four minutes when conducted without. Nonetheless, the log(ratio) of the mean values of the first and last turn pairs is not reliably different from log(1) in either the readaptation condition, $\mathrm{M}=\log (1.003), \mathrm{t}(7)=0.37$, $\mathrm{ns}$, or in the no-readaptation condition, $\mathrm{M}=\log (0.991)$, $t(4)=1.18$, ns. It appears that the aftereffect sizes reported above are fairly stable over the period of measurement.

Given the apparent stability of the aftereffect size both with and without readaptation, it is natural to wonder why the magnitude of the effect is so much greater with readaptation. It may be that the principal difference between the initial measurement condition (with readaptation) and the post-adaptation evaluation reflects a very short-term adaptation process.

\section{Experiment 2: Interocular transfer of short-term density aftereffects.}

Durgin (1992) has argued that density aftereffects demonstrate nearly complete interocular transfer when dichoptic presentation of textures allows texture patterns to be presented to a single eye with minimal subjective awareness (e.g. rivalry) of the monocularity of the presentation. Using a mirror-stereoscope to surreptitiously present textures monocularly, Durgin adapted and tested (with readaptation) one eye of eight observers and then tested (without further readaptation) either the same eye or the previously unexposed eye. Durgin found no difference in the proportional decrement of the aftereffect size whether the tested eye was the same or different from that originally exposed to adaptation stimuli. In both cases, the change in aftereffect size (log(ratio)) was by a factor of 0.5 , which closely matches the value of 0.56 in Experiment 1 . In other words, the portion of the density aftereffect which can be measured without immediate readaptation before each trial appears to transfer interocularly in its entirety under conditions of non-rivalry, though this conclusion must be tempered by the realization that the contribution of a nominally unstimulated eye to neural perceptual processing may be non-trivial. Moreover, under conditions of rivalry, Durgin (1992) reported significantly less interocular transfer of density aftereffects. 
In the present experiment we modified this interocular-transfer experimental paradigm to determine whether the short-term adaptation effects observed when observers are readapted before each measurement trial would also show interocular transfer. In our version of the study we presented the same initial adaptation stimuli dichoptically to both eyes, but then, within a single measurement session, measured density aftereffects in each eye separately, while providing readaptation textures to only one eye prior to every test trial. Such a method allows for consideration of the site of the elevated adaptation effects we observed in Experiment 1 when observers are readapted briefly before each trial. If the elevated aftereffects are present in both eyes, even when only one eye is exposed to readaptation, that would suggest that even the short-term aftereffects of texture density adaptation may be central. On the other hand, evidence that the aftereffect is reduced in the eye which is not readapted would suggest that at least some portion of the density aftereffect is monocular.

\section{Method}

The observers for this experiment were six undergraduate students and recent graduates at Swarthmore College, who were paid for their participation. The experiment was conducted using a Macintosh Quadra 610 and a ViewSonic 17 color monitor being driven at a resolution of 38 pixels/cm at $75 \mathrm{~Hz}$ (Note 3 ). The left and right halves of the monitor were viewed through a two-channel viewer built of first-surface mirrors which was carefully aligned for each observer. Each student was adapted, over a period of six minutes, to 180 pairs of textures presented dichoptically to both eyes. The textured regions subtended visual angles of approximately $10^{\circ} \times 9^{\circ}$ and were separated vertically by a distance of $5^{\circ}$. Both eyes were shown the same textures during the initial adaptation period, but during the measurement phase each eye was assessed on separate trials, and the readaptation stimuli were always presented to only one of the observer's eyes.

Adaptation was to textures of 600 dots and 100 dots presented for $1000 \mathrm{~ms}$ with an ISI of $1000 \mathrm{~ms}$. For some observers the denser texture was always above the fixation mark. For others it was always below the fixation mark. Four staircases (two for each eye) were used to assess aftereffect sizes based on the number of dots required in the dense-adapted region to appear subjectively equal to 300 and to 400 dots presented in the sparse-adapted regions. As is shown in Fig. 7, the readapted eye received three readaptation stimuli prior to each measurement trial (regardless of the eye being measured on that trial). 


\section{Dichoptic Adaptation}

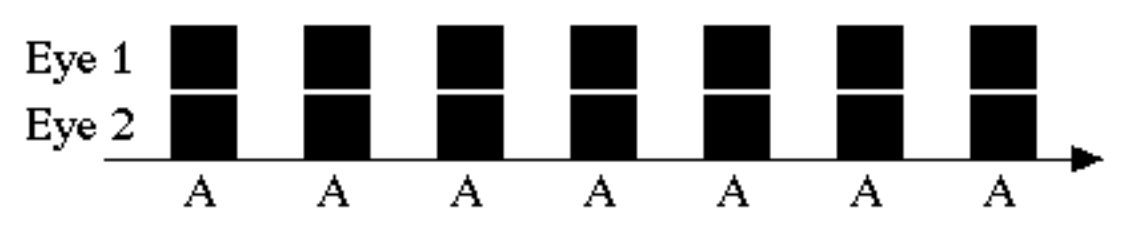

\section{Monocular Measurement and Re-Adaptation}

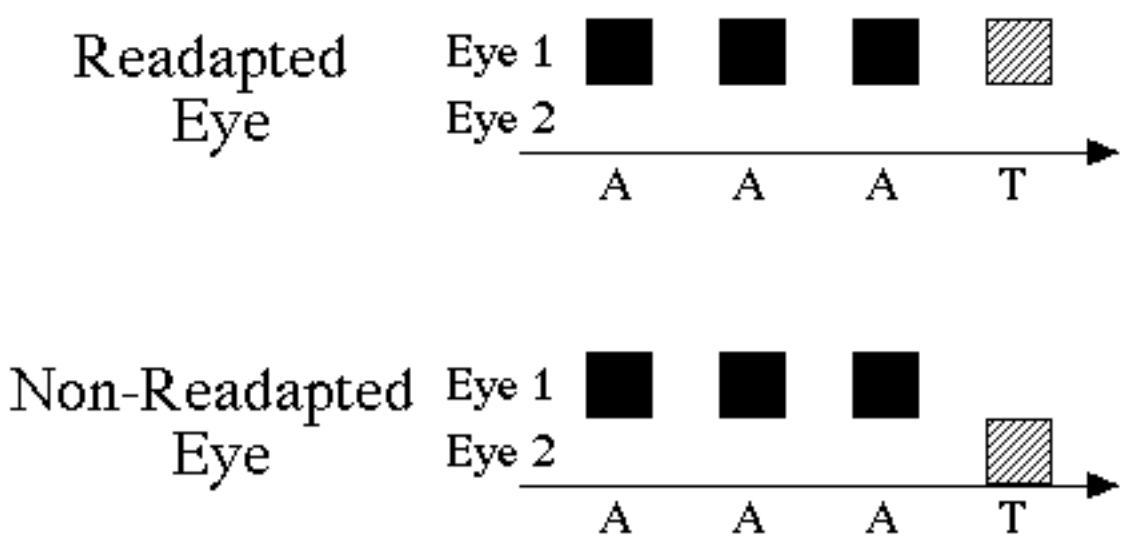

Figure 7. Schematic illustration of binocular adaptation and monocular readaptation and testing used in Experiment 2. Though both eyes are initially adapted, and each is tested (separately), only one eye received readaptation stimuli prior to each test stimulus.

\section{Results and Discussion}

The mean aftereffect sizes for each eye (Readapted and Non-Readapted) are plotted in Fig. 8. A 2 x 2 (Tested Eye $x$ Density) repeated measures ANOVA was conducted on the log(ratio) aftereffect scores computed from the PSEs for each staircase. The aftereffect measured in the readapted eye, $M=\log (1.68)$, was reliably larger than that in the eye which was not readapted, $M=\log (1.48), F(1,5)=8.89, p<0.05$. In other words, it would appear that the elevated effects found in the re-adaptation condition of Experiment 1 may be partly due to short term monocular adaptation processes. Durgin's (1992) conclusions regarding the completeness of interocular transfer of texture density adaptation therefore need be tempered by the current evidence of limited transfer of the very short-term aftereffect. 
Figure 8. Results of Experiment 2. Mean aftereffect size is plotted as a function of whether the tested eye is the one exposed to readaptation stimuli $(\mathrm{N}=6)$. Solid error bars represent the between-subject standard error of the mean. Dashed error bars represent standard error with respect to within-subject effects.

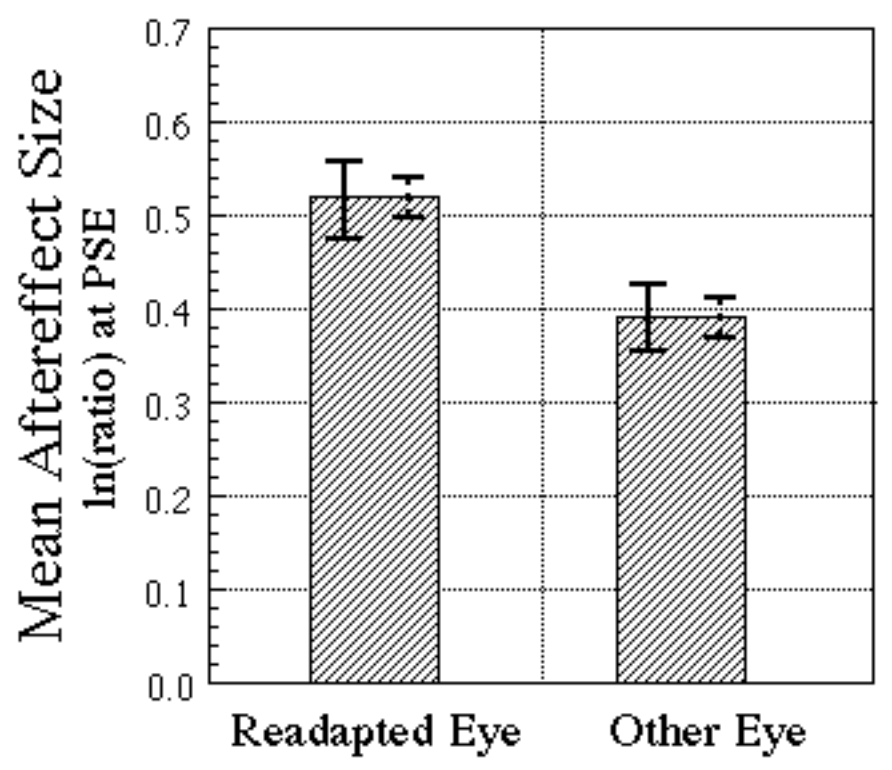

In addition, there was a reliable main effect of Density indicating that the aftereffect size computed for the 300-dot standard fields, $M=\log (1.60)$, was larger than that for the 400 -dot fields, $M=\log (1.55), F(1,5)=8.21, p<0.05$. This difference is small, and we will not try to explain it based on the present data. A trend for denser textures to produce slightly smaller aftereffect sizes was also evident in Fig. 5. It may be that a representation of density as a ratio of filled to empty surface area, rather than as dots per unit of surface area, could remove the apparent interaction of absolute density with aftereffect size. However, we also note that because the standard densities were presented in the non-adapted fields, the average number of dots in the adapted field required to match 400 dots exceeded the number of dots (600) actually used for adaptation in the present experiment.

Overall, the purpose of this experiment was to ascertain whether the short-term adaptation effects of texture density were central or peripheral in origin. Although the interocular transfer of readaptation effects was incomplete, it should be noted that the difference in aftereffect size between the readapted and non-readapted eyes in this experiment is only by a factor of 0.75 , rather than the 0.50 observed in situations where no readaptation is present at all (Durgin, 1992; Experiment 1). Although further investigations are in order, it would appear likely based on the present data that, although some portion of the measured density aftereffect is monocular, some portion (perhaps half) of even the very short-term aftereffect of texture density transfers interocularly.

\section{Experiment 3: Evidence for the retinotopic nature of texture density aftereffects.}

Clearly the visual system has 'learned' something about density by means of our adaptation procedures, but what is meant by 'learning' is not yet clear. One way of speaking of what it has learned is in terms of 'expectancies.' According to some versions of a classical conditioning approach to learning, environmental contingencies play an important role because learning is a way of adapting the organism's response to the environment. Although most visual aftereffects are retinotopic, such effects are also typically associated with specific detector units, whereas no one, to our knowledge, has proposed a density detector. Given the evidence of nearly complete interocular transfer of the longer-term density aftereffect (Durgin, 1992), it is natural to ask whether the density aftereffect is also less tied to retinal location than are other effects. To test this we used two distinct fixation positions in order to unconfound retinal and environmental coordinates.

As depicted in Fig. 9, we divided our display screen into six square regions within a black frame. This black frame surrounding the gray texture regions remained visible throughout the experiment. During initial adaptation and readaptation the adaptation textures were presented in the middle regions on the screen and the fixation mark 
appeared to the immediate left of these regions. Thus, retinal adaptation was limited to the upper right and lower right visual fields and 'world' adaptation was limited to the central pair of regions on the screen. During testing, fixation could be to either the left of right of the central region and the test stimulus could appear either to the left or right of fixation.

\section{Adaptation Stimulus}
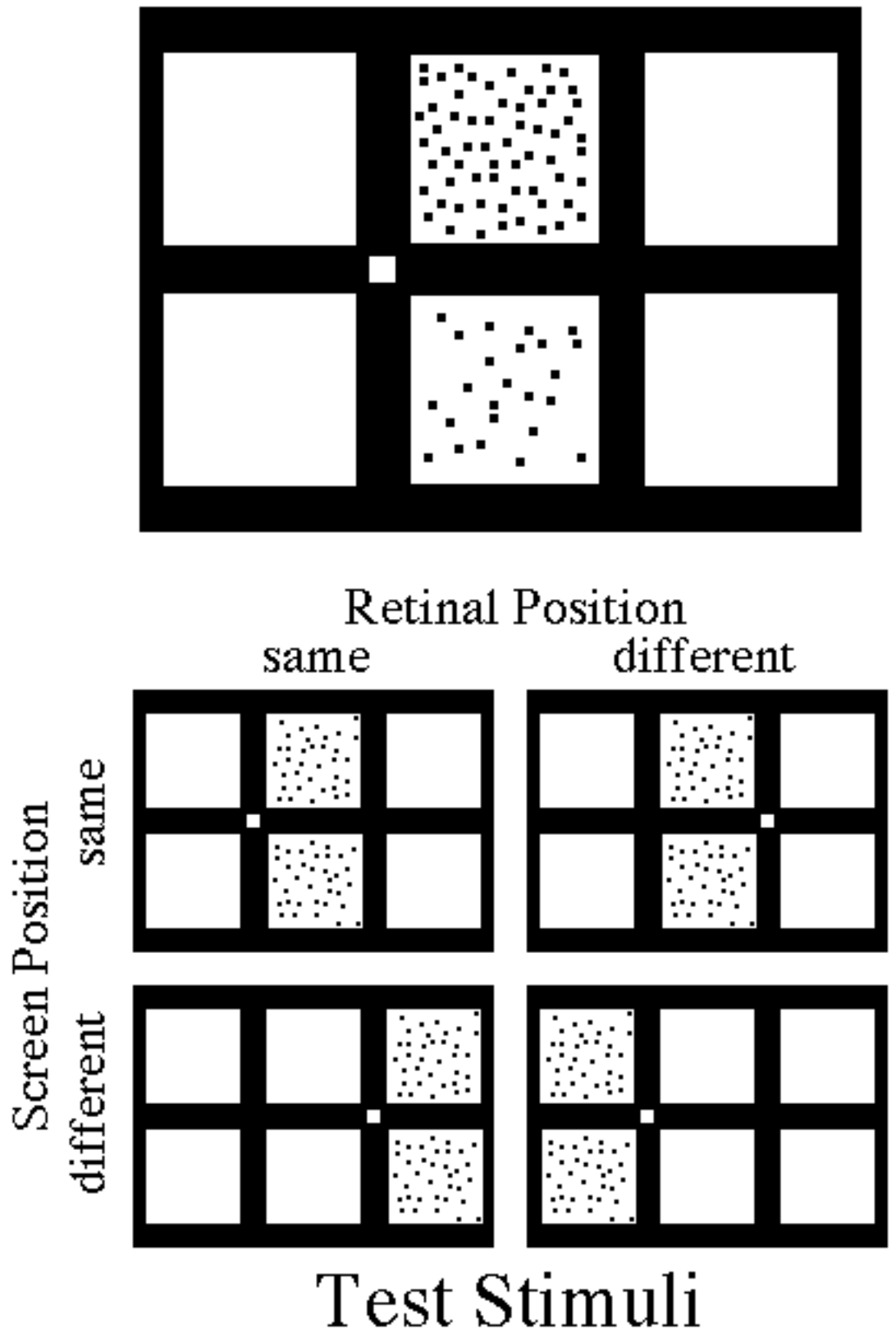

Figure 9. Schematic diagram of fixation positions and stimulus positions during adaptation (top) and those used to test whether the aftereffect was retinotopic or could be sensitive to world coordinates.

\section{Method}


The observers for this experiment were eight undergraduates at the University of Virginia, who received course credit for their participation. Adaptation proceeded much as in Experiment 1 except that the regions being adapted were smaller $\left(9^{\circ}\right.$ on a side and separated by $\left.2^{\circ}\right)$ and were presented to one side of the fixation mark.

During the measurement phase, subjects were reminded to fixate the left fixation location (only one fixation target appeared on the screen at a time) prior to each triplet of readaptation by a pair of beeps and a flash of the fixation mark. After three re-adaptation stimuli had been presented, the fixation mark for the measurement stimulus would flash and the computer would again emit two beeps. The test stimulus would be presented $1 \mathrm{~s}$ later. The observers were required to carefully fixate the visible fixation mark at all times, but actual patterns of fixation were not assessed. It should be noted that the task was fairly strenuous because of constant attentional demands.

Test stimuli were always presented in the regions either to the immediate left or right of fixation. If fixation was in the left location, then presentation to the right of fixation was identical to adaptation locations both in retinal coordinates and in screen coordinates whereas presentation to the left was dissimilar to both. But when fixation was at Position 2 , presentation to the right was consistent only with retinal coordinates whereas presentation to the left was consistent only with screen coordinates. One staircase was run for each of these four combinations of retinal and world positions, as depicted in the lower half of Fig. 9. In all four staircases, 700 dots were presented in the lower field and the numerosity of the upper field at the PSE was estimated.

\section{Results and Discussion}

Mean aftereffect sizes for each of the four stimulus types are shown in Fig. 10. A $2 \times 2$ (Retinal Position $\times$ Screen Position) repeated measures ANOVA was performed on the log(ratio) aftereffect scores. As predicted by a retinotopic assumption, aftereffect sizes were reliably greater when the retinal position of the test stimuli matched that of adaptation $(M=\log (1.65))$ than when it did not $(M=\log (1.14)), F(1,7)=34.9, p<0.001$.

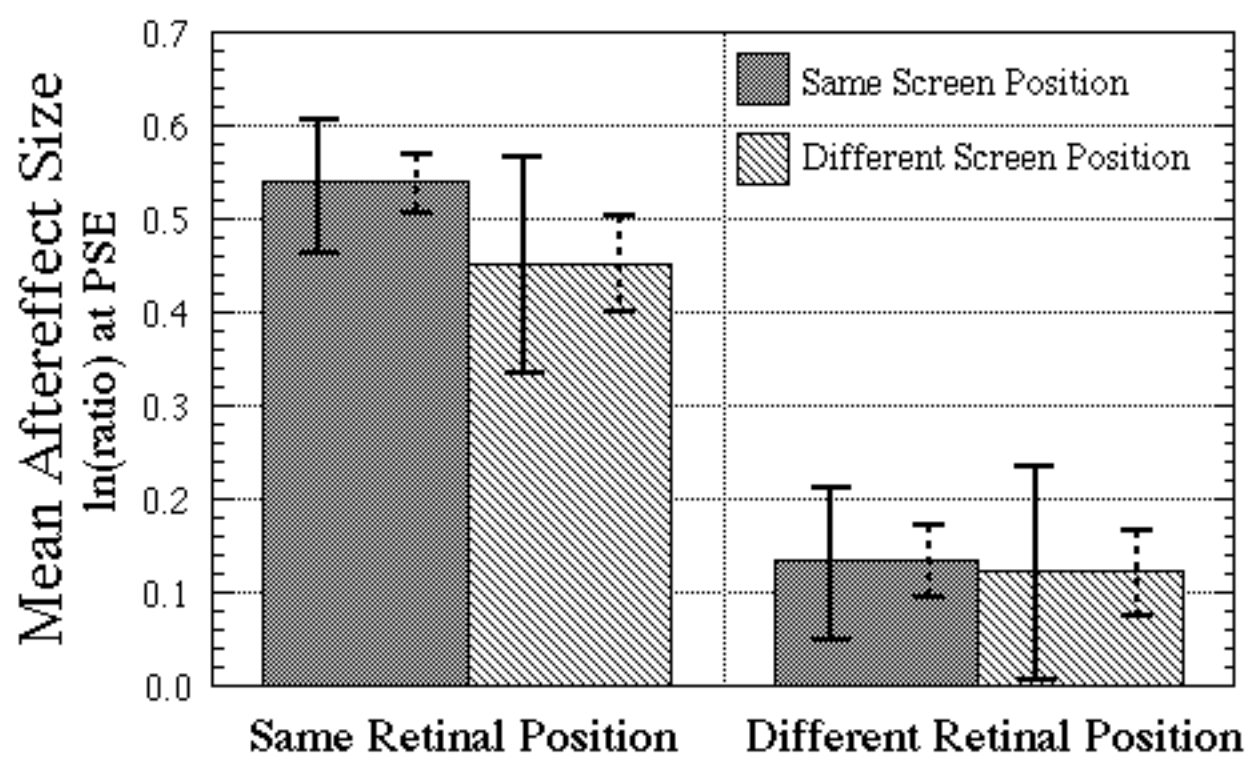

Figure 10. Results of Experiment 3. Mean aftereffect size is plotted as a function of retinal position and screen position vis a vis adaptation positions. Solid error bars represent the between-subject standard error of the mean. Dashed error bars represent standard error with respect to within-subject effects.

On the other hand, even for the test stimuli for which the stimuli were presented in retinally non-adapted locations 
the aftereffect sizes are marginally different from the presumed baseline of zero, regardless of screen position, $t(15)$ $=1.95, p=0.07$. These marginal effects may deserve further investigation, but we will make little of them here. Because fixation was not monitored, failures to refixate appropriately on each trial could produce partial adaptation of nominally unadapted retinal regions. If there is some other explanation for the effects, it may be that density perception, in general, involves large enough receptive fields to span the retinal distances between these retinally non-overlapping stimuli, or that some portion of density adaptation is sufficiently abstracted from retinal position to show effects.

Whatever the explanation for evidence of aftereffects in an unadapted retinal region, it does not appear that the effect is in any way contingent on the absolute position of the stimulus on the monitor screen, because the absolute position of the stimulus within the clearly demarcated grid on the monitor did not produce a reliable difference in aftereffect size, $F(1,7)=1.40$, ns. Any apparent reduction in size for the condition in which the retinal position was the same as in adaptation, but the screen position was not, may be attributed to the requirement to refixate on the latter trials between the adaptation and test flashes.

Although our data suggest that texture density adaptation is retinotopic, a refutation of the possibility of adapting to evironment-centered coontingencies would require the dissociation of retinal and world coordinates during adaptation (e.g. Bedford and Reinke, 1993). The present data do not encourage exploration of this possibility.

\section{Conclusions regarding the simple adaptation of texture density.}

The data presented in the first three experiments represent the demonstration of a density aftereffect that is not identical to spatial-frequency adaptation. The magnitude of the effect is quite large, though the effect appears to have a very short-term component, which is partly monocular, as well as a longer-term component which may be central. Both the very short-term and longer-term effects appear to be retinotopically localized, at least to within the two sides of the visual field. Overall, we have demonstrated the existence of a fairly well-behaved kind of visual adaptation in the perception of texture density. This adaptation effect will form the basis for our further investigations into contingent adaptation effects in the perception of visual texture.

\section{Contingent Aftereffects in the Perception of Texture Density.}

Our goals in this section are to illustrate the kinds of phenomena that theories of adaptation must be able to account for and to thereby provide a basis for later discussion of the merits of various models of contingent adaptation. We will accomplish this by an examination of various adaptation effects of visual texture density. In particular, we will describe two recently discovered contingent aftereffects of texture density, report data documenting a third, and report the results of an investigation of the effects of auditory cueing on the perception of visual texture. Before describing the contingent aftereffects of texture density, we will briefly review a few facts about contingent aftereffects.

A variety of effects have been reported in which the perceptual appearance of one visual dimension is made, by continuous pairings, contingent on the presence of some other visual dimension or feature. The classic example of this kind of effect was reported by McCollough (1965). She exposed subjects to an orange and black vertical grating and to a blue and black horizontal grating. After lengthy exposure to these two stimuli, an achromatic vertical grating will appear bluish (the complement of orange) and an achromatic horizontal grating will appear orangish. In this manner perceptual color appearance has been made contingent on orientation information.

The colors red and green have become standard for inducing the effect, but even the brightness of achromatic gratings can be substituted for color (cf., Mayhew and Anstis, 1972; Over, Broerse, Crassini and Lovegrove, 1974). Moreover, color can also be made contingent on direction of motion (Hepler, 1968) and on spatial frequency (Lovegrove and Over, 1972); motion aftereffects can be made contingent on color (Favreau, Emerson and Corballis, 
1972; Mayhew and Anstis, 1972), texture (Walker, 1972), binocular disparity (Anstis and Harris, 1974), or even direction of gaze (Mayhew, 1973); and spatial-frequency aftereffects can be made contingent on color (Held and Shattuck, 1971).

One account of these effects is to describe them as the adaptation of neurons or sets of neural units which simultaneously code both of the dimensions under question. Neural units which are jointly tuned to orientation and color, for example, have been found (Michael, 1978), as have disparity-specific motion detectors and color-specific motion detectors. However, describing contingent aftereffects as adaptations only displaces the question slightly, for what is adaptation? It is not neural fatigue, for contingent aftereffects can last for days. Moreover, there is evidence that contingent color aftereffects decay more rapidly when the visual system is active (MacKay and MacKay, 1975; Skowbo, Gentry, Timney and Morant, 1974). For example, contingent aftereffects are 'stored' during periods of sleep (MacKay and MacKay, 1974, 1975). This is not evidence against an 'adaptation' account, but rather an indication of the complexity of adaptation: Even spatial-frequency adaptation (i.e. threshold elevation) is stored during periods when the eyes are closed (Thompson and Movshon, 1978).

Evidence that McCollough effects are produced quite early in visual processing (e.g. visual area V1) include the findings that they are sensitive to retinal orientation (Ellis, 1976; cf., also, Bedford and Reinke, 1993), retinal size (Harris, 1970, 1980), and to retinal rather than perceived color (Thompson and Latchford, 1986). These effects are primarily monocular and can be demonstrated to be generated even in the absence of conscious awareness of the stimuli due to binocular rivalry (White, Petry, Riggs and Miller, 1978) or neural damage (Humphrey, Goodale and Gurnsey, 1991; Humphrey, Goodale, Corbetta and Aglioti, 1995) For a more complete review of findings regarding the McCollough effect, see Humphrey (in press).

The three contingent aftereffects of texture density we will discuss are all negative aftereffects within the visual modality which, though indicative of a sophisticated kind of adaptation, are consistent with a fairly early locus of adaptation. However, in the fourth study we will describe, we used auditory signals as cues to visual adaptation. According to a classical-conditioning model of contingent adaptation, sound should work as well as any other stimulus as a cue for contingent aftereffects. In direct contradiction to this model, we will report evidence that the use of intermodal cueing sets up a different kind of learning (a positive aftereffect) which is more like a traditional learning paradigm. Our conclusion will be that classical-conditioning models of contingent adaptation, when expressed at a molar level, do not adequately explain the constraints on contingent adaptation and contingent learning.

Though the contingent aftereffects to be reported here have not been investigated nearly as extensively as has the McCollough effect, we will suggest that our present findings, while extending the realm of contingent adaptation, are basically consistent with the kinds of conclusions indicated by these other investigators. In particular, our effects seem to be retinotopically local, and quite insensitive to global structure (cf., Broerse and O'Shea, 1995). For these reasons, our results tend not to support the (minority) view that contingent adaptation is precisely equivalent to classical conditioning (e.g. Siegel, Allan and Eissenberg, 1992). Instead, we will recommend the adoption of a more cognitive associationist view which takes functional (error correction) and informational (sparse coding) constraints on vision seriously.

\section{Texture density aftereffects contingent on color of frame.}

Durgin (in press) has reported that texture density aftereffects (for both achromatic balanced dots and for normal white dots) can be made contingent on the chromatic color of the screen surrounding the texture regions. The basic paradigm involved adapting two visual locations to equivalent numbers of dense and sparse textures, but making the presentation of a dense texture in each location contingent on a specific color being simultaneously presented on the non-textured portion of the screen. For example, on half of the adaptation trials a region to the left of fixation would be much denser than that on the right and on the other half of the trials the reverse would be true. The order of presentation of these stimuli was random, but whenever the dense texture appeared on the left, the surrounding 
screen was simultaneously turned to one color (e.g. green) and whenever the dense texture appeared on the right, the surrounding screen was turned to another color (e.g. red). During the measurement phase of the experiment separate staircases were run for trials in which a red or green frame surrounded the two (achromatic) comparison textures. (No readaptation stimuli were presented during measurement to avoid the interference of short-term simple aftereffects).

Durgin (in press) reported that this kind of adaptation produces a contingent distortion of density with a magnitude that varied with the amount of color information presented. The maximum effect sizes were obtained when both fields were entirely surrounded by color, but reliable effects were also found when only a central vertical stripe between the fields was colored, when the color was kept to peripheral regions (beyond the bounds of the rectangle defined by the two texture regions and the gray rectangular region between them), and when only the dots themselves were colored during testing. The magnitude of the effect, using balanced-dot textures was a contingent distortion of $\log (1.12)$. (Contingent aftereffect magnitudes reported here are based on a calculation of the ratio between a PSE in one context and another, such as with the green and the red surrounding frames, relative to the same standard density. As a result they are measures of combined distortion and must be halved to represent the aftereffect size in each direction.) With white dots on a black background,which have higher effective contrast, the contingent aftereffect size was as large as $\log (1.26)$ during the initial stages of measurement, but declined during the measurement procedure to $\log (1.19)$.

One of the most striking facts about this contingent aftereffect is that the color information and the texture information were in different retinal positions, yet the two apparently interact. Two other kinds of frame-contingent aftereffect have been reported previously: Potts and Harris (1975) showed that motion aftereffects could be made contingent on color and texture information presented in a surround. In addition, Siegel et al. (1992) have shown that color aftereffects can be made contingent on the lightness of a surrounding frame.

In the case of the motion aftereffect, Sharpe, Harris, Fach and Braun (1991) have argued that the effect is mediated by lateral color spreading which led to heightened activation of chromatic motion channels. But it is not clear how this explanation could be applied to the motion aftereffects contingent on the texture of the frame. It may be that these frame-contingent motion aftereffects are made possible by the lateral connections within the motion systems which lead to global cooperativity in motion perception processes.

The frame-brightness contingent color aftereffect (Siegel et al. 1992) can be explained by fairly traditional means: The McCollough effect is thought to be associated with the disappearance of the color fringes produced in the optics of the eye (or added optics such as glasses or prisms) by the differential refraction of different wavelengths of light (cf., Held, 1980). A refractive error that produced a misalignment of blue- and yellow-appearing light will produce color fringes that are blue to one side of a black bar and yellow to the other. At an edge between a dark and light surface, only one of the color fringes will be visible. Which one depends on the orientation (or contrast polarity) of the edge. Because McCollough effects are thought to be mediated by extremely local retinal processes (e.g. Broerse and O'Shea, 1995), the effects ought not to care about whether the global percept is optically consistent with a global color shift. The local indication of a refractive error in color registration would be a luminance-edge-polarity contingent color shift. In short, the color fringes that are produced by refraction (as in prism glasses, or the optics of the eye) are contingent on the luminance contrast polarity of the edges they are associated with, and it may be that the color aftereffect contingent on frame brightness is entirely determined at edges. Thus, a fairly traditional functional account of the McCollough effect can also give an excellent interpretation of frame-brightness contingent color aftereffect, so long as the frame and the central region share an edge.

Our knowledge of texture density adaptation is less well developed than that of McCollough effects (about which literally hundreds of papers have been written, cf. Humphrey, in press), but a similar functional account is worth mentioning here: The visual system's local sensitivity to fine texture details is likely to be somewhat contingent on prevailing chromatic information on the retina insofar as shorter-wavelength light is typically less well focused on the retina than is middle-wavelength light. It may be that the mechanism responsible for color-contingent aftereffects of 
texture (and even of motion) exists partly for the purpose of sorting out precisely these kinds of correlations between the kind of wavelengths of light available and the perceptual registration of texture details.

Although we mention this functional theory here, we do not mean to imply that a functional or teleological explanation is sufficient. Indeed, it may be that the global goals implied by functional accounts can be met by rather simple and quite local neural processes. Durgin (in press) argued that the contingent effect of a colored frame on the perception of texture density was probably mediated by retinal color spreading (Poppel, 1986) and by local interactions between color information and texture analyzers (perhaps where these were in closest retinal proximity). We are emphasizing functional interpretations at this point in our discussion because we believe that the consideration of functional concerns can be an excellent heuristic guide to discovering the nature of underlying processes. The power of functional considerations will be highlighted by the next contingent aftereffect we discuss.

\section{Texture density adaptation contingent on temporal sequence.}

A second, rather different kind of contingent aftereffect of texture density has been reported by Durgin and Hammer $(1994,1995)$, who studied contingent adaptation of texture perception in the domain of sequential visual inspection. They pointed out that, given the temporal mutability of perception evidenced by the ubiquity of visual aftereffects, it is surprising that our perceptions are ever accurate. In effect, there are always temporal equivalents of 'color fringes' in perception because our momentary visual processing is contingent, in some measure, on the state in which prior (as in the case of aftereffects) or even posterior (as in the case of masking) visual input has set our visual system. Thus, the question arises: Can the visual system take predictable sequential distortions into account?

There are instances of time-dependent aftereffects. For example, Anstis (1967) reported that the visual system would adapt to gradually changing luminance so that a truly unchanging gray would appear to gradually change in brightness in the opposite direction to that adapted to. In addition, Walker and colleagues have demonstrated that perceived visual or auditory duration could be made contingent on sequential presentation, (Walker and Irion, 1979; Walker, Irion and Gordon, 1981; cf., also Allan, 1984). However, we are aware of no other sequence-contingent aftereffects in which the comparison of a static spatial property such as density is affected (Note 4).

To produce these effects, Durgin and Hammer $(1994,1995)$ adapted observers to sequentially presented pairs of balanced-dot textures which differed either in the density or in the luminance contrast of the dots. They then measured PSEs for density and for brightness using sequentially presented textures with readaptation prior to each test trial. (The temporal parameters during adaptation were $200 \mathrm{~ms}$ of one texture, $200 \mathrm{~ms}$ ISI and then $200 \mathrm{~ms}$ of the second texture. During testing the ISI was varied between 100 and $400 \mathrm{~ms}$ with little affect on the resulting aftereffects.) A schematic illustration of the adaptation paradigm is shown in Fig. 11.

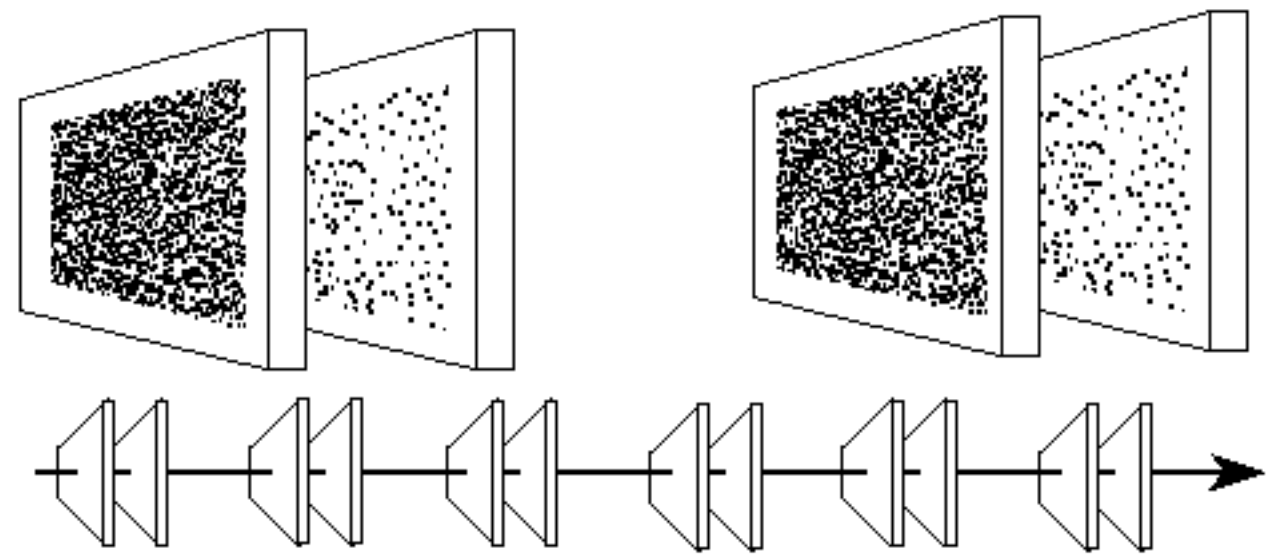


Figure 11. Schematic illustration of temporal pattern of sequence-contingent adaptation. Each adaptation stimulus consisted of a pair of textures presented sequentially which always differed in a particular way (e.g. here the first is always denser). After adaptation to the situation depicted here, the second of two sequentially presented equally dense textures will appear denser than the first.

Durgin and Hammer found that adaptation to sequentially presented textures that differed in density produced density aftereffects contingent on the order of presentation: If adaptation had been to sequences of dense followed by sparse balanced-dot textures, then objectively equal textures presented sequentially would appear to go from sparse to dense. Adaptation to luminance contrast differences between equally dense textures produced parallel aftereffects of perceived texture element brightness. Notably they found that the manipulation of texture contrast did not produce any contingent distortion of perceived density, though adaptation to density differences did produce a weak contingent effect on perceived texture brightness.

Although Durgin and Hammer offered no explicit neural model of their effect, they did look at two kinds of constraints that any such model must satisfy. First, they found that variations in temporal parameters, such as stimulus onset asynchrony (SOA), that are normally quite significant in motion perception, did not seem to greatly affect either the density or the brightness sequence-contingent effects (Durgin and Hammer, 1994). In addition, investigations of interocular transfer of these sequence-contingent effects indicated that the brightness effect is more monocular (shows less interocular transfer) than the density effect (Durgin and Hammer, 1995). The brightness effect may be related to Anstis's (1967) luminance-ramp aftereffect, which is monocular.

The most striking fact about these effects is that they seem superficially quite incommensurable with the standard spatial and retinotopic models of adaptation effects. They could be made more commensurable if they were regarded as the adaptation of change detectors (or expansion and contraction detectors, Regan and Beverly, 1978), but Durgin and Hammer $(1994,1995)$ produced the sequence-contingent density aftereffect with SOAs as long as $600 \mathrm{~ms}$, which is too long for first-order motion processing, and the balanced dots textures used are poor inputs to motion processing (Gilden et al., 1990). Moreover, the introspective experience of observers doing the task is one of comparison of static entities, not a registration of change. It may nonetheless be incorrect to think of the processing of apparently temporally-discrete stimuli as truly discrete, because the validity of introspection is questionable.Somehow or other a neural reverberation of the first texture may serve as a context in which the second appears, or perhaps the two textures define an event, the registration of which is affected by contingent adaptation.

Durgin and Hammer (1995) argued that it is easy to imagine that these kinds of sequence-contingent adaptations are quite useful in normal perception: Precisely because our visual processing apparatus is so adaptive, it ought to have a way of canceling out those dynamic fluctuations in response (which might be termed short-term aftereffects) that it can learn to predict. In normal perception the visual system is given ample opportunity to observe and become normalized to simple adaptation effects from the sequential registration of many different kinds of visual stimuli (e.g. saccades allow the rapid successive viewing of distinct stimuli). It may be that by simulating a repeated biased sequential comparison, normal calibration processes can be made to produce the kinds of sequence-contingent aftereffects Durgin and Hammer observed both for texture density and texture brightness.

\section{Texture density distortion contingent on orientation of elements.}

One approach we have taken to trying to track down the basis for the contingent adaptation of texture density perception is to look at somewhat more traditional cues for contingent adaptation. The visual system is known to be very sensitive to orientation, and contingent interactions between orientation and perceived surface color are the basis for the McCollough effect. We have therefore tried to determine whether the orientation of texture elements can serve as a cue upon which perceived texture density can be made contingent.

There are a number of reasons to believe that this could occur. The texture discrimination learning described by 
Karni and Sagi (1991) is specific to the orientation of the background texture elements, rather than of the foreground elements that were to be judged. Interpreted within an 'energy' model of texture perception (e.g. Bergen and Adelson, 1988; Malik and Perona, 1990), this suggests that their subjects may have developed visual mechanisms that helped to 'filter out' the specific background texture they had been trained on (or adapted to). Such an energy interpretation would be consistent with the retinotopic effects found by Karni and Sagi (Note 5). Moreover, such local effects could also arise in the short term from orientation-specific threshold elevation (Blakemore and Campbell, 1969) if density itself were perceived by means of stimulus 'energy' (normalized to local contrast).

Because we were interested in comparing adaptation to globally defined stimulus differences to local contingent adaptation, we devised two types of contingent adaptation stimuli. (Interest in this distinction was partly motivated by consideration of current classical conditioning models of adaptation for which global stimuli are considered the inputs, such as that of Siegel et al., 1992). For one set of observers, each adaptation stimulus consisted of a dense texture on one side and a sparse texture on the other. The side on which the denser texture appeared was contingent on whether the textures were composed of vertical or horizontal line segments, as shown in Fig. 12A. For the second set of observers, the same kinds of textures were presented on each side of fixation, but no density differences were ever presented. Instead, the dense horizontal texture and the dense vertical texture were presented simultaneously, and likewise for the sparse textures, as illustrated in Fig. 12B.

If texture density adaptation occurs with regard to a global relationship between two regions, then the pairing of orientation and a density difference ought to produce a greater effect than merely presenting equal densities of differently oriented elements in different locations. However, if the contingent adaptation observed depends only on retinotopically local stimulation, then no difference between the two adaptation conditions should arise.
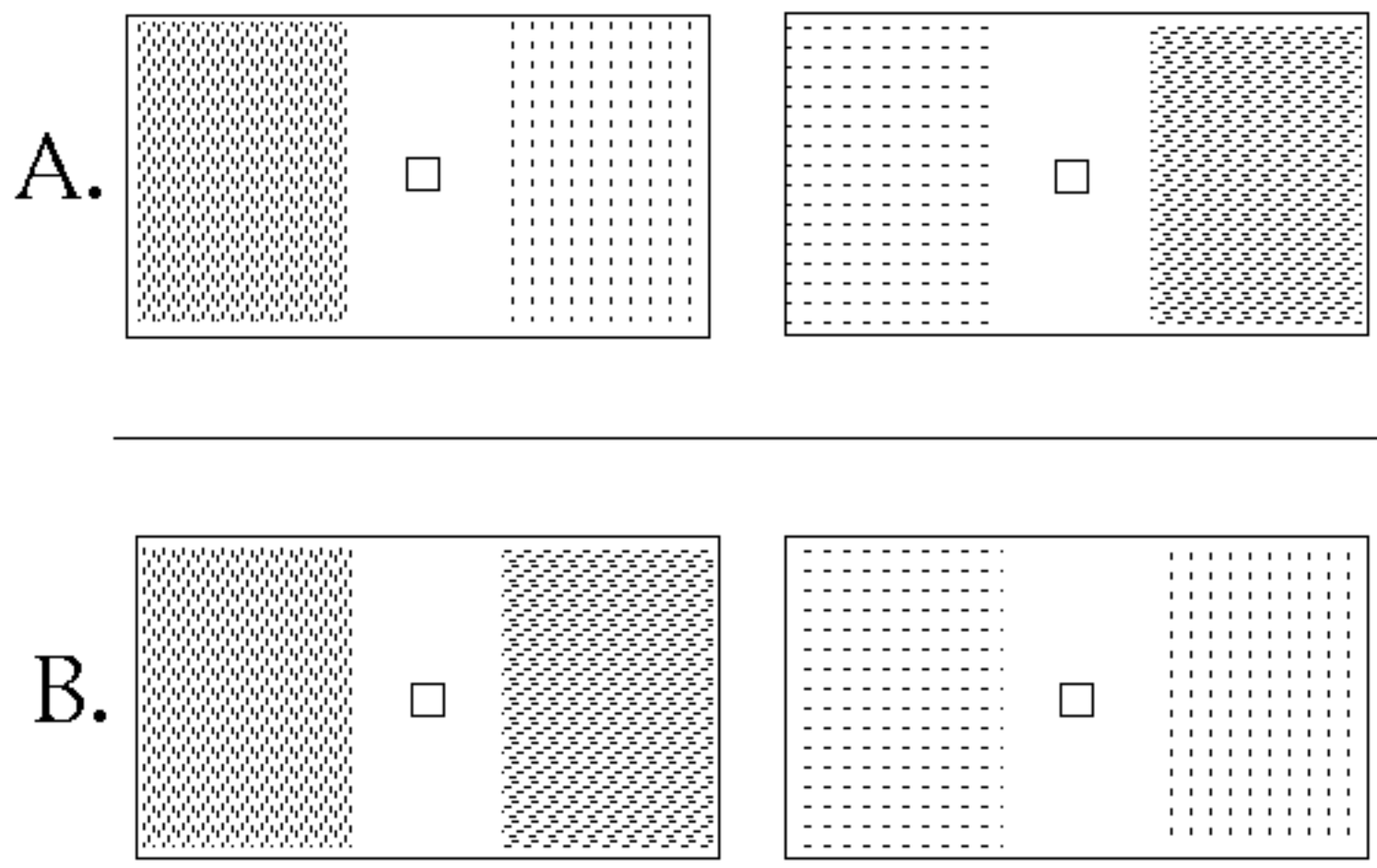

Figure 12. Schematic diagrams of pairs of adaptation stimuli in which texture element density is locally correlated with texture element orientation. In A, element orientation and a global difference in density are correlated. In B, local element density is still correlated with local element orientation, but global density differences are never presented. Actual textures used in experiments were not periodic. See Figs 13 and 14. 
orientation-specific distortions of perceived density. Instead, these element-orientation-contingent density aftereffects depend entirely on differential local stimulation, consistent with almost any model of retinotopic texture analyzer adaptation. In Experiment 5 we will provide evidence suggesting that the Fourier discriminability of the differently oriented texture elements may play an important role in determining the strength of contingent adaptation.

Durgin and Proffitt (1991) originally reported some evidence that texture density aftereffects seemed to be relatively texture-element non-specific. They tested for aftereffect transfer from white-centered balanced dots to blackcentered balanced dots and vice-versa. Balanced dot textures, whether black or white, have similar power spectra, however. Although the size of the texture-element contingent aftereffects reported here is quite small compared with the simple density aftereffect, the current experiments, using textures with different orientations, do not support their original contention.

\section{Experiment 4: Orientation contingent density adaptation is local not global.}

The schematic layout of a texture-element-contingent density aftereffect adaptation paradigm is illustrated above in Fig. 12. Panel A of the figure illustrates a case where the leftmost of two simultaneously presented textures is denser when the textures are vertical and the rightmost is denser when horizontal; Panel B illustrates a case where the vertical dense textures always appear on the left as before, but are now accompanied by a horizontal dense texture on the right. Note that the actual experimental textures were newly generated on each trial and were not periodic like those shown.

The two panels of Fig. 12 represent the two adaptation conditions used in the present experiment. The global adaptation condition is that shown in Panel $A$, in which global density differences are presented. In the local condition, shown in Panel B, global density differences are never presented, though the adaptation stimuli are the same locally as in the global condition.

\section{Method}

In this study, 16 University of Virginia undergraduates were adapted to contingencies between local density and texture orientation in one of two conditions. Either (1) element orientation was identical for each of the two textures within each adaptation trial and local density was contingent on orientation (Global Correlation condition, Fig. 12A) or (2) the density of two adaptation textures was equal within each adaptation trial and local orientation was contingent on density (Local Energy condition, Fig. 12B). Particular pairings between orientations and locations were randomized between subjects as was the assignment to condition.

The purpose of including both conditions was to test whether a texture-contingent density aftereffect would be greater if global density differences were present during adaptation. The stimuli presented locally in the two conditions were entirely equivalent; only the pairing of stimuli was varied. For comparison purposes, both paradigms were tested with pairs of textures in which element orientations were the same, and judgments were gathered concerning perceived density.

\section{Stimuli}

The textures were generated by scattering a specified number of oriented bars against a black background. Each bar was a 2- by 12-pixel white rectangle within a 4- by 24-pixel black region in which no other bars could be placed. New random textures were generated on each trial of adaptation and testing.

The textures were generated and presented and data was collected on a Sun 3/60 computer with color monitor (resolution of 40 pixels/cm). Texture areas were 240 by 320 pixels and were displaced 60 pixels to the left and right 
of the center of fixation. (At a viewing distance of $43 \mathrm{~cm}, 30$ pixels is approximately equal to 1 deg visual angle.) Each texture was presented for $200 \mathrm{~ms}$.

\section{Adaptation}

Subjects received the same local adaptation in both conditions. There were 500 adaptation trials, all prior to any measurement, representing 250 exposures to: (1) a sparse (54 element) vertical-element texture on side A, (2) a sparse horizontal-element texture on side B, (3) a dense (600 element) vertical-element texture on side $B$, and (4) a dense horizontal-element texture on side A. The side that was defined as A (left or right) was varied randomly between subjects. What differed by condition was whether exposure to (1), for example, was simultaneous with exposure to (2) (Local Energy condition: LE) or to (3) (Global Correlation condition: GC). Adaptation textures were presented at a rate of approximately one per second though this value was increased by as much as $200 \mathrm{~ms}$ in the LE condition when dense textures were generated for both fields.

\section{Measurement}

Following adaptation, four PSEs were measured by a staircase method (without readaptation). One PSE was measured for each texture element orientation (horizontal or vertical) at each of two standard densities (240 and 400 elements). The standard density was always presented on the left and the variable field was always on the right side (though this fact is not evident to the observer who simply chooses whichever side appears denser).

\section{Results and Discussion}

The log(ratio at PSE) scores were analyzed in a $2 \times 2 \times 2$ (Condition x Texture Orientation x Standard Density) mixed design repeated measures ANOVA. As anticipated, the PSE for texture density was indeed contingent on the orientation of the elements used: More elements were required on the right side during testing when the orientation being tested was that which had been denser on the right during adaptation, $F(1,14)=9.21, p<0.01$. The magnitude of the effect was quite small, however relative to the simple density aftereffect. Because Texture Orientation was a within-subjects variable, the effect of orientation can be represented by a difference score between orientation conditions. The overall mean texture-orientation-contingent difference calculated in this way was $\log (1.067)$. Note that because this value represents the sum of distortion in both directions, this magnitude of aftereffect is extremely small relative to the measure of the simple density aftereffect (without readaptation) in Experiment 1.

However, contrary to the predictions of the global pattern hypothesis, the log(ratios at PSE) in the GC condition, $M=$ $\log (1.071)$ did not differ reliably from those in the LE condition, $M=\log (1.061), F(1,14)<1.0$, ns., nor did the effect of Texture Orientation differ as a function of Condition, $F(1,14)<1.0$, ns. In short, the orientation-contingent effect found here did not depend on the presence of density difference during adaptation and appears to be entirely attributable to local adaptation effects.

Finally, as in our other experiments, we again found that the magnitude of contingent adaptation effects (expressed as logarithms of ratios) were not dependent on the magnitude of density being tested, $F(1,14)<1.0$, ns.

In summary, the results of this experiment show that the perceived density of a local texture region can be made contingent on the orientation of the texture elements that compose the texture. This contingent aftereffect does not depend on a density difference being present during adaptation, but only on the presence of differential local stimulation. In fact, it is consistent with a simple adaptation of orientation-tuned neurons that might contribute to the coding of density, though the nature of the adaptation itself remains unknown. 


\section{Experiment 5: Testing the importance of orientation discriminability.}

In Experiment 4 we used white line elements presented on a black background and found a fairly weak contingent aftereffect. Such stimuli have very high contrast so the weak effect was somewhat surprising, because it has been shown that texture density aftereffects from white dots on a black background produce strong density aftereffects (Durgin, 1995b), and frame-contingent aftereffects (Durgin, in press). However, the orientation information in such textures may be relatively weak because low-pass filtering will reduce such textures to unoriented blobs. To test whether improved Fourier texture discriminability would heighten the orientation contingent aftereffect found in Experiment 4, we designed luminance-balanced lines (Carlson et al. 1984) for use as texture elements and compared orientation contingent aftereffects with balanced lines to similar aftereffects obtained with matched, but unbalanced lines.

Representative illustrations of the texture stimuli used and their 2D Fourier Power Spectra are shown in Fig. 13. The use of balanced elements produces a texture with band-pass characteristics. As a result, it can be seen in the Fourier spectra that the orientation-specific distribution of energy at the center of the spectra is better defined for the balanced texture. This gain in orientation-specificity makes sense because the texture formed by these elements becomes less detectable by low-frequency blob detectors and more similar to Gabor-like patterns which are thought to be fairly closely matched to human pattern analyzers (see, for example, Graham, 1989).

\section{Method}

Twenty-two University of Virginia undergraduates participated for partial course credit. Half were assigned to the balanced-line condition and half to an unbalanced-line condition. The design within each of these conditions was similar to the design of Experiment 4: some subjects were adapted in a Global Correlation condition and others in a Local Energy condition. For each observer, measurements were collected at two densities for each of two test orientations.

The balanced-line elements each consisted of a 2- by 10-pixel black center with a 1-pixel wide bright annulus which was contained within a 'protected' region, 5- by 20 -pixels. The luminances of the annulus and of the background gray were adjusted so that the total luminance contribution of each balanced line was exactly the same as the background that it displaced (Note 6 ). The mean luminance of all balanced textures was $11.8 \mathrm{~cd} \mathrm{~m}-2$. The textured regions of the screen were increased to two 300- by 400-pixel areas displaced 60 pixels from the center of fixation. As before, dense adaptation texture contained 600 elements, sparse textures contained 48 . A portion of a representative dense texture is shown in Fig. 13. 

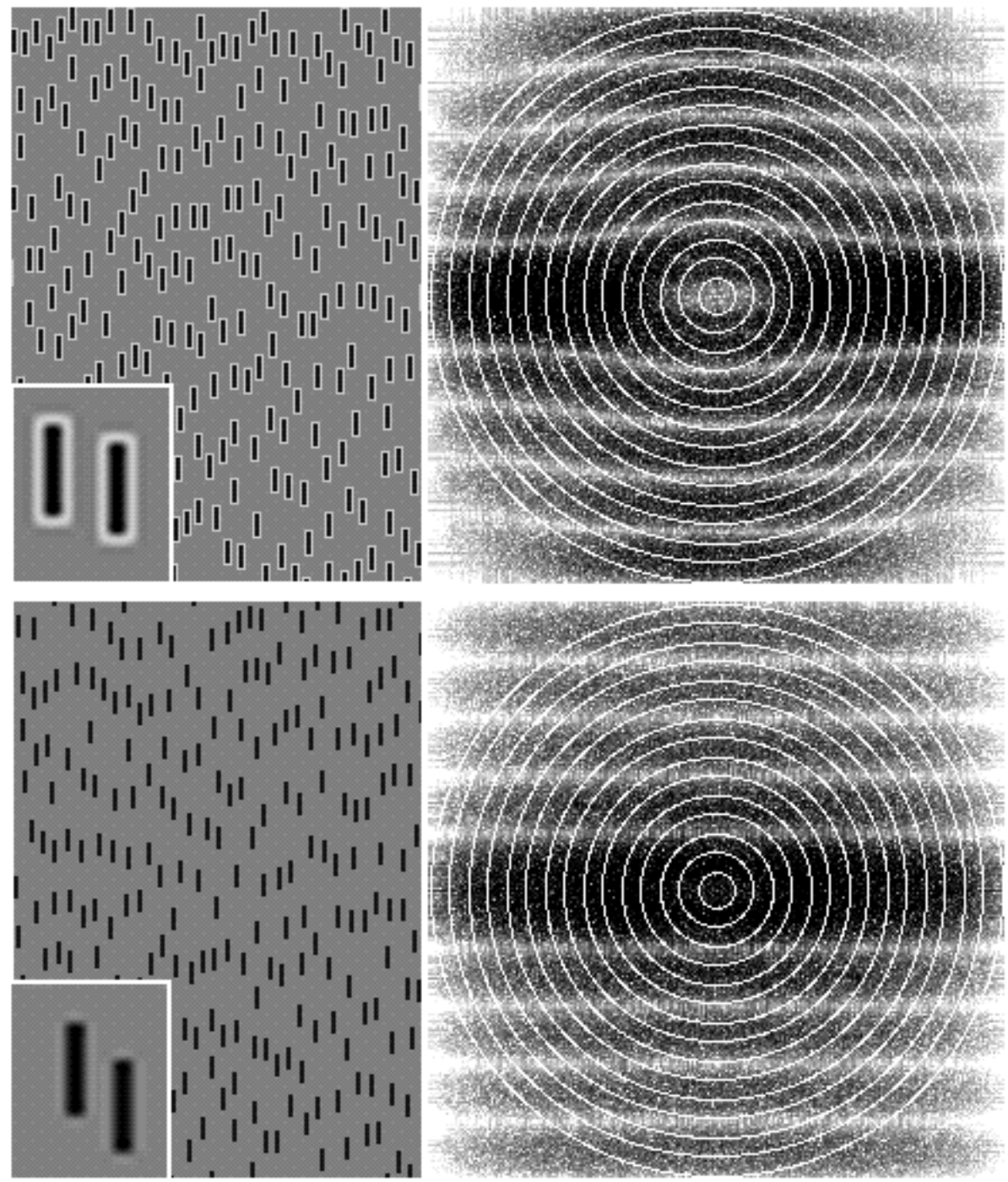

Figure 13. The power spectra (right) of textures composed of luminance-balanced lines (top) or of unbalanced lines (bottom). In these polar plots, spatial frequency and orientation are plotted as $r$ and $q$, respectively, and amplitude (or contrast energy) is denoted by darkness. The innermost ring represents a spatial frequency of $1 \mathrm{cpd}$; each concentric ring represents an increase in spatial frequency by $1 \mathrm{cpd}$. It can be seen that the power spectrum for an unbalanced line texture is fairly homogenous out to $3 \mathrm{cpd}$, whereas that for a balanced texture is demonstrates substantial orientation differentiation at 1-2 cpd. In the near periphery, the visual system must rely on lower spatial frequencies. These balanced textures have greater orientation specificity than do the unbalanced.

In Experiment 4 the ISI between stimuli during adaptation covaried slightly with the density of the stimuli. This was of particular concern in the LE conditions where some stimuli contained two dense textures (which take longer to generate) and others contained two sparse ones. To eliminate this potentially confounding influence of temporal spacing during adaptation, 8 randomized versions of each of the four adaptation textures were generated before each experimental session. These images could then be loaded in random order at a constant rate during adaptation, unaffected by the number of elements. Stimulus duration in the present experiment was $500 \mathrm{~ms}$ and the ISI between adaptation stimuli was $800 \mathrm{~ms}$. Adaptation and measurement proceeded otherwise just as in Experiment 4.

\section{Results and Discussion}

The $\log ($ ratio at PSE) scores were analyzed in a $2 \times 2 \times 2 \times 2$ (Element Type $\times$ Adaptation Condition $\times$ Element 
Orientation $x$ Standard Density) mixed design repeated measures ANOVA. As expected, there was a reliable effect of Texture Orientation in the predicted direction, $F(1,19)=23.4, p<0.0001$. However, there was also an interaction between Texture Orientation and Element Type, $F(1,19)=9.06, p<0.01$. The data for the two Element Types were therefore analyzed separately.

In the Balanced Lines group, there was a reliable effect of Texture Orientation in the predicted direction, $F(1,9)=$ 15.1, $p<0.01$. The mean contingent aftereffect size (computed as in Experiment 4) was log( 1.19). Thus, using balanced lines we find a reliable aftereffect of texture density contingent on the orientation of the texture elements. It should be noted, that, as in Experiment 4, there was no evidence that the effect of orientation was modulated by the adaptation condition (GC or LE) for this group, $F(1,9)<1.0$, ns. Thus, this effect can be explained as arising from local exposure to dense oriented textures, so that adaptation to global differences in density are not required.

For the Unbalanced Lines group, there was no reliable effect of Texture Orientation, $F(1,9)=1.67$, ns, though there was a trend in the appropriate direction. The aftereffect sizes for each kind of element type are plotted in Fig. 14, along with the effect size for Experiment 4.

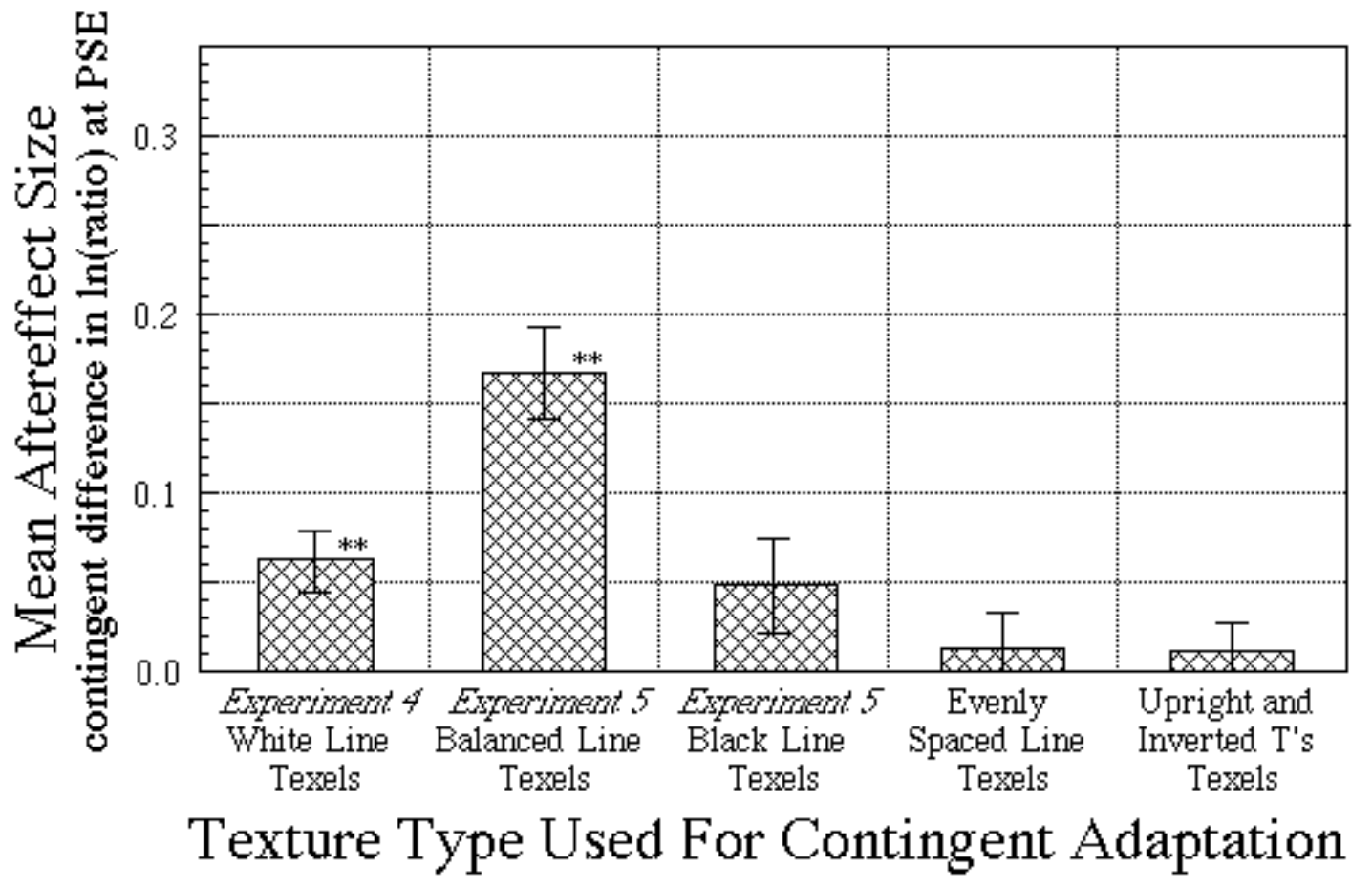

Figure 14. Mean aftereffect sizes for Experiments 4 and 5 are shown alongside data from two other experiments described briefly in the text (texture stimuli for Experiment 5 are shown in Fig. 13; other texture stimuli are shown in Fig. 15). Contingent aftereffect strength is greatest for balanced lines. Error bars indicate standard error of the mean.

The results of the present experiment replicate the essential findings of Experiment 4: The texture-contingent density aftereffects appear to result from purely local exposure to texture rather than to globally available texture differences.

\section{Texture-Contingent Adaptation and Fourier Texture Discrimination.}

The results of Experiment 5 provided evidence that differential adaptation effects (contingent adaptation) were greater for balanced-line textures than for the unbalanced ones, and it is clear from Fig. 14 that the contingent aftereffect evidenced with balanced lines exceeded that obtained even in Experiment 4 with high-contrast white-onblack textures. As mentioned above, the orientations of balanced-line textures are more discriminable at low spatial 
frequencies than are their unbalanced counterparts. This can be seen by direct comparison of the power spectra of balanced and unbalanced textures, as shown in Fig. 13. We have overlaid a white polar grating indicating the spatial frequencies represented by the spectra. The inmost ring is 1 cycle per degree (cpd) and each additional ring represents the next higher integral value of cpd. It can be observed that the central three rings are homogenous, for the unbalanced texture, but not for the balanced texture. The power spectra of balanced lines are orientationally asymmetric at spatial frequencies as low as $1 \mathrm{cpd}$, whereas those of the unbalanced textures are not well defined until $3 \mathrm{cpd}$. This outcome may at first appear counterintuitive because the original purpose of balanced lines (i.e. Carlson et al., 1984) was specifically to reduce the low-spatial frequency components. However, because these lines are balanced globally for luminance and not locally, they actually resemble a dipole stimulus (there is 'more annulus' at the ends than in the middle), and DeValois and Switkes (1980) have shown that dot-field stimuli consisting of oriented doubles (essentially dipoles) produce differential orientation-specific threshold elevation for oriented gratings. On almost any account, the strength of a contingent aftereffect (differential adaptation to the two distinct texture types) ought to depend in part upon the visual discriminability of the cueing stimuli. It is therefore not surprising that Fourier discriminability seems to predict aftereffect strength.

We have undertaken two further experiments to determine how reducing the Fourier discriminability of stimuli would affect success in generating the effect. The experiments will not be described in detail here, though they only differed from what we have already described in the textures used. The texture stimuli used and their Fourier transforms are shown in Fig. 15, Panels A and B. It can be seen that for the stimuli in Panel A, the elements are spaced apart somewhat (each element takes the place of a virtual square). This spacing has the consequence of utterly eliminating any orientation specificity in the low spatial-frequency regions of the power spectra, as can be observed from the homogenous central disk of the power spectra of Panel A. The power spectra of Panel B are essentially identical to each other. T's and upside-down T's have the same power spectra, and thus so do textures composed of them. Six subjects were adapted using stimuli like those in Panel A; eleven were adapted using stimuli like those in Panel B. No evidence of contingent aftereffects was found in either case. The mean contingent aftereffect scores for these two types of stimuli were $\log (1.015)$ and $\log (1.012)$ respectively. These were less, in both cases, than the associated standard errors of the mean. Although it is possible that a larger number of observers may have produced a reliable difference, the effect, if any, would probably be quite small. In summary, two attempts to induce element-contingent density aftereffects with textures that did not differ in their low spatial frequencies were unsuccessful.
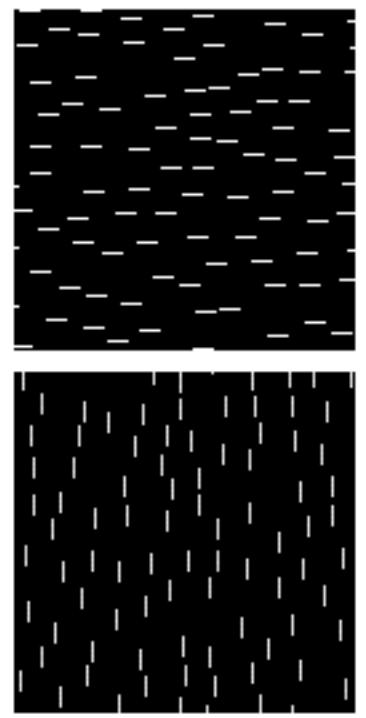
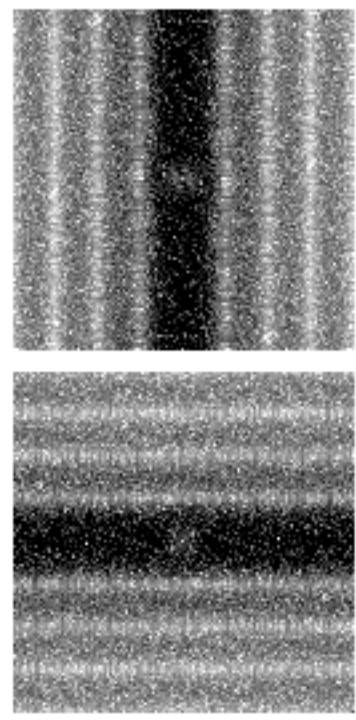

A
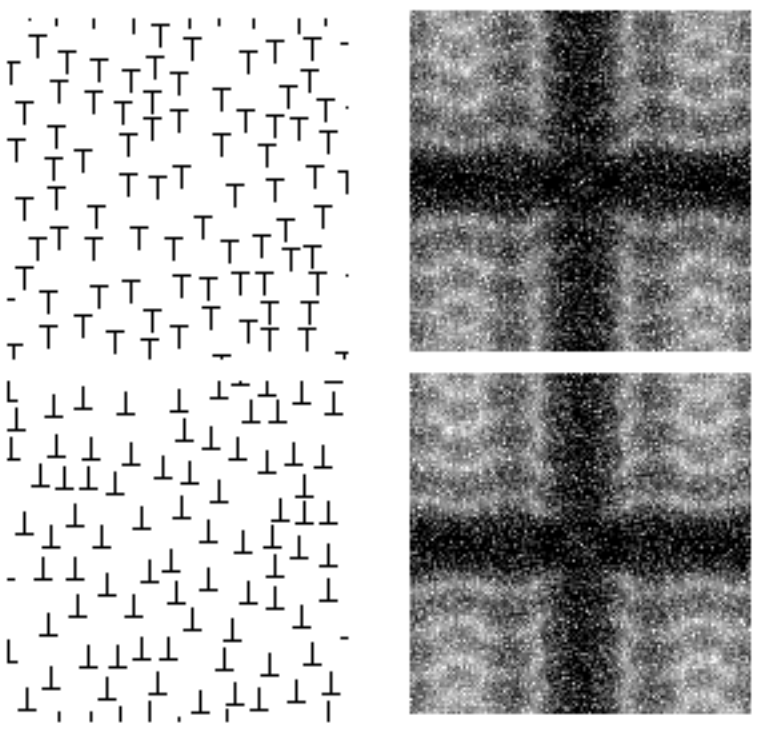

B 
Figure 15. Sample textures and power spectra of stimuli for which we have found no evidence of orientationcontingent aftereffects. Lines in Panel A are embedded within virtual squares, as are the Ts in Panel B. In both cases, the power spectra demonstrate little discriminability (orientation specificity) at low spatial frequencies.

This finding leaves open two distinct possibilities. (1) The effect is actually one of reduced sensitivity to the specific texture elements following adaptation. Such a loss of sensitivity might result in the texture appearing less 'numerous' or dense as a result of changing the input to the texture density registration process. (2) Alternatively, the result may be understood as reflecting an adjustment of the gain of the output of the density system that is yoked to characteristics of the local texture.

The first alternative appears superficially more parsimonious: We already know that sensitivity to specific orientations and spatial frequencies decreases following adaptation to them, and there is evidence, with periodic stimuli, that brightness and density can interact perceptually (Mulligan and MacLeod, 1988). However, although the two accounts cannot be completely dissociated by means of the experimental evidence that we have reviewed thus far, the first account appears to depend on density and contrast energy being interchangeable with respect to adaptation. Durgin and Hammer (1994; cf., also, Durgin, 1995a) were able to differentiate density and brightness adaptation in sequential perception, so it appears unlikely that the simplest version of the sensitivity hypothesis (Explanation 1) is correct. Moreover, even if visual texture density is computed as a function of total energy scaled to peak contrast, then it is unclear how threshold elevation can give an adequate account because the registration of both of these quantities should be affected by it.

The general pattern of results here is quite consistent with the supposition that local texture density aftereffects can be made contingent on the kind of retinotopically local texture information presented. Though further studies of interocular transfer may be required to tie this effect to the orientation-specific texture learning reported by Karni and Sagi $(1991,1993)$, the present evidence is at least consistent with the existence of a connection between texture registration, texture discrimination and aftereffects in the perceived density of texture.

Why should there be orientation-contingent texture density aftereffects? There are many kinds of answer to this question, but one particular kind of answer strikes us as potentially fundamental. This is the concept of coding efficiency based upon visual expectancy. Many have argued that a sparse visual code is a more effective way to process visual stimuli than is massive parallel action of the entire visual cortex (Field, 1994; Foldiak, 1990). A sparse visual code can be created by having highly tuned, specialized analyzers. If too many analyzers respond to the same stimulus information, that is inefficient and may call for adjustment of the analyzer response, which could be achieved by lateral inhibition between analyzers in a sort of competition for coding rights (Barlow and Foldiak, 1989; Kohonen and Oja, 1976). Several models of adaptation have been proposed that rely on the build-up of inhibition between simultaneously activated units (e.g. Tolhurst, 1972). Barlow and Foldiak (1989; Barlow, 1990), in particular, have proposed an inhibitory or 'anti-Hebbian' model to account for contingent aftereffects.

The orientation-contingent density aftereffect described here has been shown to be responsive to local density information but not to global differences between separate textured regions. This finding is consistent with the postulation of a very early site of texture adaptation, as hypothesized for spatial-frequency adaptation, texture discrimination learning and contingent color aftereffects. If the effects are construed as orientation-specific changes in the responses of filters in primary visual cortex, they may be related to the texture discrimination learning reported by Karni and Sagi $(1991,1993)$. The learning Karni and Sagi have investigated was specific to the background texture employed, but not to the foreground texture, suggesting that a kind of local texture filter may be involved (Sagi, 1994). Admittedly, the contingent texture aftereffects we have described were measured only in the short term and therefore do not indicate any permanent alteration in visual processing as did the learning experiments of Karni and Sagi. 


\section{Texture density aftereffects contingent on auditory pitch?}

The final experiment we will describe is concerned with the effects of adapting to the correlation of relative texture density with an auditory signal. The experiment was inspired by the claims of classical conditioning theorists of contingent adaptation. A strict conditioning account might well predict an equivalence of auditory and visual cues for inducing conditioned visual responses (Note 7). However, such an outcome would be extremely surprising from the point of view of most recalibration or neural adaptation accounts. On the other hand, informative correlations between sight and sound are known to sometimes have an assimilatory influence on perception. For example, in the McGurk effect, the auditory perception of a phoneme can be altered by the simultaneous visual presentation of someone uttering a different phoneme (McGurk and MacDonald, 1976). Note that the distortion observed in the McGurk effect is toward rather than away from the normally paired auditory and visual stimuli. De Sa (1994) has shown that such effects as the McGurk effect may arise because the simultaneous and correlated activation of different modalities can normally help to constrain the interpretation of information within each modality.

\section{Experiment 6: Auditory cues produce 'anomalous' contingent aftereffects.}

The general design of the present experiment is similar to those reported above. The principal change is that the density differences to which subjects are adapted are correlated with an auditory cue, as depicted schematically in Fig. 16, rather than a visual one. To our knowledge, there are no prior reports of intermodal contingent adaptation, nor is the effect found in the present experiment a true contingent aftereffect. A contingent bias is found in subjects' density judgments, but the bias is in the opposite direction from the effects we have described above.
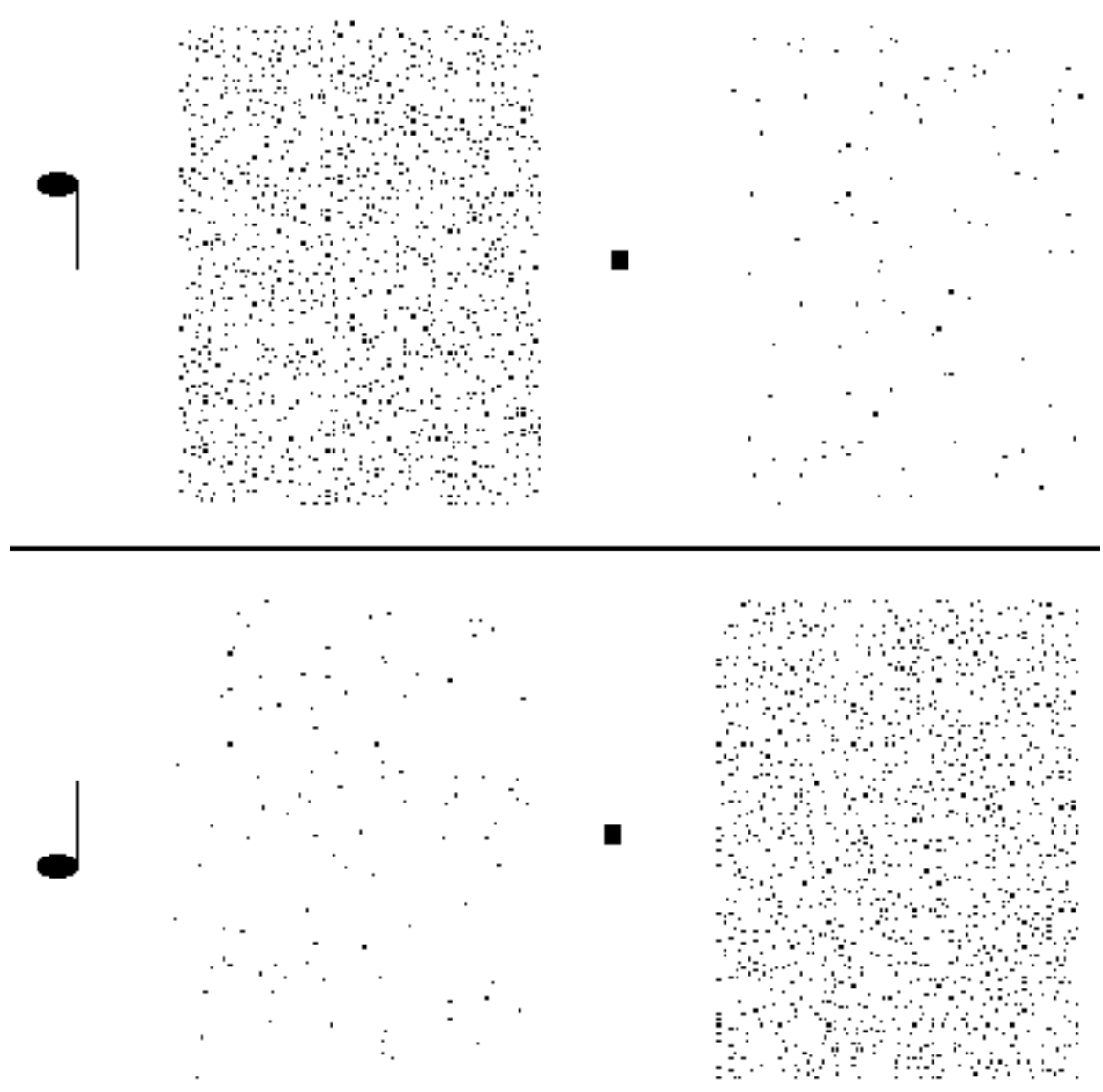

Figure 16. Schematic illustration of adaptation paradigm for Experiment 6. On each adaptation (learning) trial a high or low tone is presented while a texture pair appears about a central fixation mark. For each subject, auditory pitch 
is perfectly correlated with the position of the dense texture.

\section{Method}

Twenty-four undergraduate students at the University of Virginia received course credit for their participation. The experiment was run on a Sun SparcStation 2 computer with audio capability and an 8-bit RGB display monitor, 1152 by 900 pixels, with a resolution of 40 pixels $/ \mathrm{cm}$. Subjects were seated at a viewing distance of $61 \mathrm{~cm}$ (distance measured once the subject was seated comfortably). The room was darkened.

Two rectangular regions of the screen were used to present scattered-dot textures. The regions were 240 by 320 pixels (approx. 6 by $8 \mathrm{deg}$ ) and were offset from the center of the display by 60 pixels (approx. 1.5 deg) to the left and right. A small fixation mark was constantly present at the center of the display. During adaptation, subjects were exposed to 180 brief (128 ms) flashes of adapting stimuli with an ISI of approximately $1000 \mathrm{~ms}$. The onset and offset of each flash coincided with the onset and offset of a computer-generated buzzing tone. In each adaptation flash, balanced-dot textures were presented in the two regions, one was dense ( 25 dots deg- 2 or 1200 dots) and the other sparse (2 dots deg-2 or 96 dots). The auditory pitch of the simultaneously presented tone was perfectly correlated with the side (left or right) on which the denser texture appeared. The tones used were $500 \mathrm{~Hz}$ and $74.3 \mathrm{~Hz}$. The pairing of each tone with a particular side was alternated between subjects. The screen was entirely gray $(12 \mathrm{~cd} \mathrm{~m}-2$ -- equiluminant with the adaptation textures) except for the fixation mark, during the ISI. The flashes were brief to reduce the possibility of unintentional eye-movements during stimulus presentation. Figure 16 depicts the adaptation stimuli schematically.

The point of subjective equality (PSE) of density for textures presented in the two test regions was assessed for two levels of density (400 and 600 dots, or 8.33 and 12.5 dots deg-2) in the presence of each of the two auditory pitches. Subjects made forced-choice decisions about which of two simultaneously flashed textures was denser. Measurement was accomplished by the same modified staircase procedure described in previous experiments. Apart from their density, test textures had the same spatial and temporal parameters as those used in adaptation.

Observers were tested individually. Each was instructed to use the fixation mark during both the adaptation and measurement phases. Observers were informed that they would first be asked to pay attention to the screen for several minutes without responding (the adaptation phase) and would then be required to judge the relative texture density of the two textured regions of the screen (measurement phase). Subjects were explicitly informed that this was a visual task and were told to be sure to make the judgments visually. They were warned that tones would accompany the stimuli, but that they should do their best to ignore the tones. After the presentation of all of the adaptation stimuli, the screen stayed blank until the subject pressed a button to go on to the measurement phase. No recovery period was used between adaptation and measurement because simple adaptation effects produced by the exposure to the stimuli could not account for any sound-specific bias.

During the measurement phase, subjects indicated whether the left or right texture field appeared denser on each trial by pressing either the left or right mouse button. There were 80 measurement trials, on average, over a period of about three minutes.

\section{Results and Discussion}

A 2 × 2 (Standard Density $\times$ Auditory Pitch) repeated measures ANOVA was performed on the log-transformed ratios at PSE. There was a reliable main effect of Auditory Pitch in the direction opposite to other contingent aftereffects, $F(1,23)=6.75, p<0.05$, indicating that in the presence of a pitch previously associated with a dense texture on one side, that same side now required fewer dots in order to be chosen as the denser field. As was also expected, there 
was no effect of standard density, $F(1,23)=2.07$, ns.

The pattern of results demonstrated here indicates that the perceptual comparison of dense textures can be biased by prior contingent pairing with auditory pitch (or some other aspect of the auditory signals we used). The direction of the bias is opposite to that found in all of the previous experiments, and is consistent with a learned association between pitch and location of higher density. Though this is but a single example, it seems to greatly weaken the already weak case for a strict conditioning account of contingent aftereffects: If classical conditioning is intended to predict the results of contingent pairing between perceptual dimensions, it cannot, without further modification, predict why the bias found here (with an auditory cue for a visual stimulus) is in a direction opposite to that found when visual cues are used.

An analysis of the aftereffect size scores of individual subjects (i.e. the difference of logarithms scores, such as reported for previous experiments) revealed an extremely broad range of scores. The average aftereffect size was $\log (0.91)$, or -0.093 , which, except for the sign, is comparable to other contingent aftereffects reported for texture density. However, the inter-subject variability was large: the standard deviation was 0.17 . Though fifteen of the subjects demonstrated the anomalous aftereffect with a score of -0.05 or less, there were seven who demonstrated a 'normal' aftereffect with a score of 0.05 or greater. This diversity of results may indicate that more than one kind of process was at work. What appears clear from these data are that the use of auditory pitch as a cueing stimulus does not simply result in the same kind of distortion as does temporal sequence, frame-color, or texture element orientation. It is worth noting that the results of this experiment are quite robust. A pilot experiment using black and white textures produced nearly identical results -- which was initially disconcerting to us because it 'didn't work' in the manner we had originally expected it to.

Instead of producing error-correction, the nature of the adaptation experience produced a clear positive association between pitch and spatial location. Many observers agreed that they experience the pitches as emanating from the position of the denser texture on each adaptation trial. Many also felt that their attention was subsequently drawn to the location signaled by the concurrent pitch (i.e. that based upon the earlier pairings). Since audition and vision clearly do join up in our experience of the perceptual world (Gibson, 1966), and spatial location is coded in both modalities, this is a sensible place for interaction to occur. If spatial attention was cued by audition in this manner, the attended texture may have appeared denser in consequence simply of being attended.

In follow-up experiments to this work, Harp, Lewis and Durgin (1995) have shown that visual attention may be involuntarily drawn to the visual locations signaled by pitches originally associated with those locations during adaptation. Harp et al. (1995; Durgin and Harp, 1995) had observers adapt to sound and density pairings much like those of Experiment 6 . They have shown that subsequent to such adaptation, observers can respond more quickly to a balanced dot stimulus presented to one side of fixation if it is cued by the pitch that was paired with denser texture on that same side during adaptation. It may be that the learned auditory capture of visual attention demonstrated by Harp et al. is sufficient to produce either a perceptual or judgmental bias toward seeing or choosing one side as denser than the other in a manner contingent on auditory pitch.

\section{Theories of contingent adaptation and visual learning.}

One of the goals of this article is to place our findings of contingent density adaptation within the context of visual learning, because we wish to demonstrate that there are important parallels between simple and contingent texture adaptation and other forms of learning in the perception of visual texture. We will therefore preface our theoretical discussion of contingent adaptation with a brief look at some relevant studies of visual learning. Thereafter we will evaluate several theories of contingent adaptation in order to demonstrate what we believe are the most important current ideas about contingent aftereffects. Once we have developed a model of simple and contingent adaptation we will discuss what the empirical findings presented in this article may imply about texture perception and visual learning. 


\section{Visual Learning}

Although there have been a number of reports of visual learning that is quite sensitive to the specific stimulus parameters involved (e.g. McKee and Westheimer, 1978; Ramachandran and Braddick, 1973), the recent work of Karni and Sagi (1991) represents a particularly interesting example of visual learning in the domain of texture perception. They examined visual learning in a texture discrimination task which involved detecting the orientation of a small texture-defined region within a briefly presented and rapidly masked stimulus. (The region is defined by oriented bars but its orientation is not the same as theirs.) The dependent measure used to assess learning was change in the minimum stimulus onset asynchrony between stimulus and mask that was necessary to reach some criterion of performance.

Karni and Sagi found evidence of visual improvement at the task which was retinotopic, monocular (but see Schoups et al., 1995) and specific to the orientation of the background texture. Karni and Sagi also reported that the learning did not occur with mere exposure when attention was devoted to another task. Sagi (1993) has further reported that there is a consolidation of this very specialized visual learning during sleep. Observers will continue to demonstrate local, monocular and orientation-specific learning which can improve not only within a session, but also between sessions if REM sleep intervenes. Ahissar and Hochstein (1993) have shown that attentional factors influence this learning, but Karni and Sagi (1993) found that, once established, the learning that occurs in these contexts can be retained for at least 2-3 yr and probably indefinitely. This kind of learning is clearly a long-term improvement in visual texture discrimination.

A second important kind of learning is error-correction or recalibration. In order for straight lines to appear straight, and curved lines curved, our visual system must be properly calibrated (Andrews, 1964). Andrews proposed that there must be error-correction units within the visual system in order to maintain the perceptual uniformity of the geometry of space.

There are at least two kinds of empirical evidence of this sort of recalibration. One kind concerns vernier acuity, the ability to visually determine whether, for example, two line segments are collinear. McKee and Westheimer (1978) demonstrated stimulus-specific improvements in vernier acuity judgments with practice. More recently, Poggio, Fahle and Edelman (1992) have described a simple network model for task-specific vernier acuity learning. They reported human psychophysical data indicating that learning is rapid, monocular, retinotopic, and orientationspecific, and task and stimulus specific, and show that their network can mimic all of these properties. Note that the work of Poggio et al. can be understood as task-specific learning of the kind described in the previous section, but also as a mechanism for maintaining an accurate representation of Euclidean collinearity.

The second kind of evidence of relevant structural change was reported by Wolfe and O'Connell (1986) in the form of a very long-term tilt aftereffect (measurable several days after induction). The methods of induction and measurement are quite similar to a vernier task because Wolfe and O'Connell used pairs of nearly vertical gratings, as illustrated in Fig. 17, which were either parallel (collinear) or at an angle to one another. It is therefore possible to construe their aftereffect as a recalibration of the visual perception of parallelism or collinearity. 


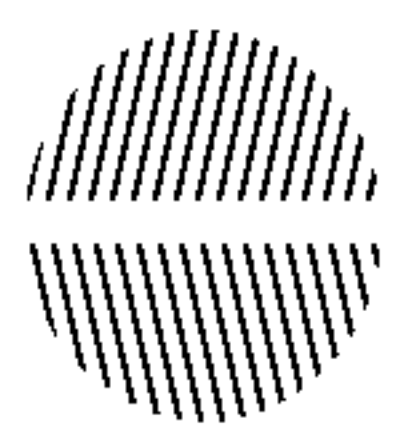

Adapt
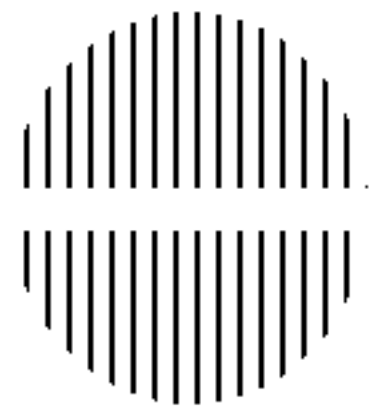

Test
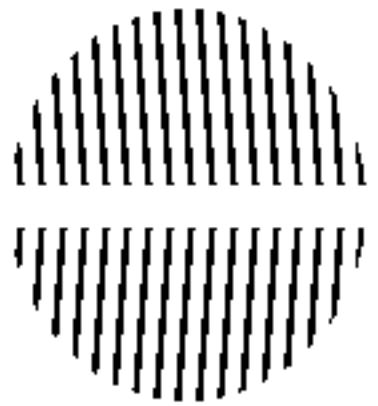

Perceive

Figure 17. Illustrations of adaptation and test stimuli used to produce long-term tilt aftereffects (Wolfe and O'Connell, 1986). Note that the task used to assess a tilt aftereffect in this case is to judge whether two sets of lines are collinear and parallel -- which is very similar to a variant of a vernier acuity task, such as those studies by Poggio et al. (1992).

\section{Recalibration and error correction as a model of contingent adaptation.}

Recalibration accounts have also been applied to contingent adaptation (e.g. Held, 1980). Indeed, the relationship betwen recalibration and contingent adaptation has been extensively discussed in a recent volume of this journal (cf. Broerse, Shaw, Dodwell and Muir, 1994; Dodwell, 1994; Humphrey, Gurnsey and Bryden, 1994). Dodwell and Humphrey (1990, 1993; Humphrey, in press) have spelled out a recalibration account of the McCollough effect explicitly in terms of error-correction. They cite Andrews (1964), as well as Helson's (1964) theory of adaptation level (cf. also Gibson, 1933). The idea of error correction that they suggest requires an Error Correction Device (Andrews, 1964) which tracks correlations between form and color and works to remove them. In Dodwell and Humphrey's version of the error-correction hypothesis they have chosen to have the system be responsive to global vector fields corresponding to the Lie transformation group (i.e. circular and hyperbolic gratings). Current evidence that effects are local rather than global suggest that this property of the model requires revision (cf. Humphrey, in press; see also Broerse and O'Shea, 1995). However, this portion of the theory is inconsequential to the main hypothesis which is that a contingent aftereffect is a both a normalization process and an error-correction process.

Anstis (1975) has discussed a related notion, describing contingent adaptation as a means of eliminating cross-talk between visual channels. The essential idea in Anstis's proposal is that the visual system must be diligent at keeping its visual channels from leaking into each other: Correlations between channels are evidence of a leak and are corrected. A similar recalibration account of contingent adaptation has been discussed by Wolfe (1990) following on the Wolfe and O'Connell (1986) demonstrations of the long term tilt aftereffect.

Recalibration accounts offer a functional (or in Marr's, 1982, terms a 'computational') account of what the visual system ought to be doing (Dodwell and Humphrey, 1990, 1993; Humphrey, in press). After all, the input receptors for daytime vision are cones which simultaneously code both intensity and color. It must be the business of the visual system to code the various dimensions it extracts as cleanly as possible, but this can only be accomplished if the circuitry involved is forced to keep the various kinds of information sorted out. Whether one describes this as the elimination of cross-talk between channels (Anstis, 1975) or as error-correction and normalization (Dodwell and Humphrey, 1990), the essential problem of keeping channels of information segregated is a real one and the effects observed in contingent adaptation appear to be the kind that such accounts ought to predict.

One difficulty with recalibration accounts of contingent aftereffects is that they sometimes appear somewhat cumbersome because they propose entities such as Error Correcting Devices (ECD) which are described as if 
separate from the original encoding machinery. An extreme example from a recent proposed variant of these kinds of explanation suggests that contingent aftereffects are based on assumptions of object constancy (Bedford, 1993, 1995). In Bedford's scheme, contingent adaptation depends on the visual system recognizing that the cue information (e.g. orientation in the case of the McCollough effect) is embedded in the same object viewed from a different perspective (e.g. a grating viewed vertically or horizontally). This high-level recognition must then provide feedback to the encoding system to inform it of a need to contingently adjust its gain on the assumption that objects should appear the same (e.g. in color) from all perspectives. Although the idea that some high-level process is important to contingent adaptation may well be correct, there already exist a large number of empirical counterexamples to the object constancy hypothesis in the form of McCollough effects produced with locally orthogonal, but topographically distinct forms (Broerse and O'Shea, 1995; Dodwell and O'Shea, 1987; Dodwell and Humphrey, 1990; Emerson, Humphrey, and Dodwell, 1985; Humphrey, Dodwell and Emerson, 1985, 1989; McCollough, 1994; Yasuda, 1978). Indeed, it appears to us that even the adaptive elimination of optically induced color fringes which may have originally inspired the McCollough effect are difficult to explain in object-constancy terms, though easily handled by less elaborate assumptions.

Other error-correction accounts have proposed simpler, but perhaps similarly unnecessary assumptions. For example, Dodwell and Humphrey (1990; see also Wolfe, 1990) suggest that the visual system's ECD assumes that a zero correlation should exist between color and orientation. Why should this be so? Durgin and Hammer (1994, 1995) have assumed that ECDs could correct for biasing errors that might normally arise in the sequential registration of brightness and density information, but give no further reason for why this ought to happen. For this reason, these kinds of account appear to remain at the level of functional description with varying degrees of face validity.

\section{Classical conditioning accounts of adaptation}

If some of the assumptions of certain error-correction models seem arbitrary, an attractive alternative position has been to describe these effects as resulting from a single, arguably non-arbitrary process of classical conditioning (e.g. Murch, 1972, 1976; Siegel et al., 1992). More general associative accounts have also been proposed (e.g. Harris, 1980; Mayhew and Anstis, 1972; Montalvo, 1976), but the currently dominant advocates of the classical conditioning position speak of learned associations between stimuli as if the processes of perception were governed quite simply by classical conditioning. The most common formulation of the classical conditioning explanation for the McCollough effect, for example, holds that the UCS is color, the CS is orientation, and that the CR is color adaptation.

Though frequently dismissed as misguided or demonstrably wrong (e.g. Dodwell and Humphrey, 1990; Humphrey, in press; McCarter and Silver, 1977; Savoy, 1984; Skowbo, 1984, 1986; Stromeyer, 1978) the classical conditioning account of contingent adaptation (e.g. Murch, 1972) retains staunch advocates (e.g. Allan and Siegel, 1986, 1993; Siegel and Allan, 1992; Siegel, Allan, and Eissenberg, 1992, 1994) and a number of important supportive findings based on predictions from the Rescorla-Wagner (1972) model of classical conditioning (Siegel and Allan, 1985; Sloane, Ost, Etheridge and Henderlite, 1989; Westbrook and Harrison, 1984). There remain advocates of more precisely formulated associative theories, as well (Barlow, 1990; Montalvo, 1976), but these theories differ from the strict classical conditioning account in that they are concerned with associations that are modified between neurons, not between stimuli (though a less strict conditioning account could be similarly maintained).

Why does a classical conditioning approach help our understanding of contingent adaptation? The primary appeal for many has been parsimony. Associative accounts have often been contrasted with accounts that hypothesize the adaptation of detectors sensitive to both dimensions, and it has seemed to some that the proliferation of multidimensional detectors is ungainly (Harris, 1980). A second appeal has been the longevity of the effects (Jones and Holding, 1975), which also argued against simple neural fatigue. The problem with these imputed advantages is that almost any theory that is not based on the fatigue of individual detector units can deal with both concerns admirably. There are only a few dozen different kinds of contingent adaptation (cf., Durgin, in press, for an overview), and 
longevity may apply even to simple aftereffects (e.g. Wolfe and O'Connell, 1986).

What the classical conditioning approach has been good at predicting about contingent aftereffects are certain kinds of interactions derived from the Rescorla-Wagner model of associative learning (i.e. blocking and overshadowing, Siegel and Allan, 1985; Sloane et al., 1989; Westbrook and Harrison, 1984). Although the findings reported do not prove that the Rescorla-Wagner model is the correct one (Sloane et al., 1989), they do suggest that some kind of associative principles may be involved.

What the classical conditioning approach has been poor at predicting is the neural/informational locus of adaptation and, for related reasons, the characteristics of the stimuli that can induce contingent aftereffects. The account does not explain why most of these effects are monocular, retinotopic and sensitive to retinal rather than perceptual characteristics of stimuli (e.g. retinal color, size and orientation). It could, if it chose, appeal to 'preparedness,' but perhaps that has been avoided as introducing a degree of arbitrariness. Indeed, the efforts of classical conditioning theorists (e.g. Siegel et al., 1992) to demonstrate that a broad variety of stimuli (i.e. not just simple visual features) can support contingent adaptation have invariably led to controversies over just how retinotopic the effects are. For example, Allan, Siegel, Collins, and MacQueen (1989) reported color aftereffects contingent on specific printed words and a failure with non-words, but others report that they obtain these results only under conditions of steady fixation, which suggests that it is retinotopically local edges that matter (Humphrey, Skowbo, Symons, Herbert and Grant, 1994). Similar apparent misunderstandings concerning the very local character of contingent color aftereffects have been observed for other non-simple stimuli (cf., Broerse and Grimbeek, 1994; Broerse and O'Shea, 1995; Humphrey, Herbert, Symons, and Kara, 1994; Siegel, et al., 1992, 1994).

Another pitfall of the classical conditioning approach is illustrated by the results of Experiment 6 , in which we demonstrated a positive bias in perceived density following adaptation contingent on auditory pitch. Although the basic paradigm in that experiment is formally similar to the others, the process that was engaged is evidently of a very different kind (and more like what most people think of as classical conditioning). With respect to clear constraints on what kinds of stimuli ought to produce contingent adaptations, the classical conditioning theory falls far short of error-correction theory.

One way of conceptualizing the situation is to argue that, viewed from within the primary visual cortex, something very like classical conditioning (i.e. Hebbian modification of synaptic strength) may be occurring. But viewed at a more molar level the resulting process looks very like modular error-correction. Moreover, because the stimuli that are involved appear not to be those that enter into consciousness (e.g. wavelength rather than perceived color, Thompson and Latchford, 1986; cf. also Houck and Hoffman, 1986; Humphrey, Gurnsey, and Fekete, 1991; Thompson and Travis, 1989; White, Petry, Riggs, and Miller, 1978), to describe contingent aftereffects as the classical conditioning of color and orientation, for example, is potentially very misleading -- especially for those who are naive about visual processing.

Consider the results of Experiment 6, in which an auditory pitch produced a positive bias in judgments of density. Expressed in terms of biological decision making or information theory, it is quite sensible to treat strong correlations in the environment as information which can be applied to the process of making a judgment. If density was unambiguously greater on the right side whenever the higher pitch sounded, it is rational to expect that the presence of an unambiguous high pitch could later influence a fairly ambiguous decision about the relative densities of two textures that are quite similar in density.

An explicit theory concerning the efficacy of combining auditory and visual inputs has been developed by de Sa (1994; Ballard and de Sa, 1994). She suggested that human category learning within a modality can be vastly improved by using temporally coincident patterns of activity in different modalities to modify decision rules (categories) within each modality. The case she considered involved correlations between auditory and visual inputs to the interpretation of spoken language. She showed that a simulation of an unsupervised neural network could perform almost as well as a supervised network (i.e. one given feedback during learning) if it used temporally coincidental activity between modalities to provide feedback to both. 
The function of learning about multi-modal correlations can be clearly understood, but the function of contingent adaptation is less clear, if not for error-correction (Dodwell and Humphrey, 1990). Siegel and Allan (1992) have invoked the concepts of homeostasis and conditional compensation, but do not explain why they should apply to the known contingent aftereffect pairings and not to others. In contrast, an advocate for an error correction model, such as Dodwell and Humphrey's, can assert that only intramodal contingent adaptation will demonstrate negative contingent adaptation because it is only intramodal information that is confounded in receptor inputs (e.g. wavelength and form information are detected by the same receptors).

In summary, the classical-conditioning view of contingent adaptation appeared at first to be more parsimonious than error-correction models, but closer examination suggests that it requires just as many, if not more, arbitrary qualifications in order to count as an explanation. In the end, it may turn out to be a good description of some aspect of the underlying mechanisms of contingent adaptation, but its usefulness will probably depend on the development of explicit neural models of adaptation, like Montalvo's (1976).

\section{Adaptation and information processing}

Both error-correction and associative learning seem to have some merits, but the one conception that has gone largely unanalyzed is McCollough's original formulation of her effect. McCollough's (1965) explanation was that she was investigating the color adaptation of edge detectors. This has commonly been taken to mean something like the fatigue of double-duty (color and form) detector units, but a rather different interpretation is required if the term adaptation is examined more closely.

In Helson's (1964) classic exposition of Adaptation Level Theory, he begins by explaining what he means by adaptation to distinguish it from alternative conceptions. For many psychologists today, as when Helson wrote, visual adaptation is still regarded primarily as indicative of neuronal fatigue. This conception is that of the physiologist, and is supported to some degree, in the case of aftereffects, by neurophysiological evidence. For example, Barlow and Hill (1963) demonstrated a decline in the response of motion sensitive cells with steady exposure to motion. Note, however, that such a result, in itself, does not indicate the cause of the decline. A number of investigators have argued from psychophysical evidence that the build-up of inhibitory effects are partly responsible for featural adaptations (e.g. Blakemore, Carpenter and Georgeson, 1970; 1971; Tolhurst, 1972), and neurophysiological evidence supports this (e.g. Vautin and Berkley, 1977). It is also worth noting again that threshold elevation from spatial-frequency adaptation, for example, is maintained during periods of visual inactivity but declines rapidly when the visual system is active (Thompson and Movshon, 1978; cf. also MacKay and MacKay, 1974). This is consistent with the idea that visual adaptations are the result of an active process in the visual system, rather than a failure of its material substrate. In summary, as Helson argued, perceptual adaptations are not identical to neuronal fatigue, and the two concepts ought not to be confused.

Before turning to the consideration of Barlow's model of contingent and simple adaptation (1990; Barlow and Foldiak 1989) which is based on inhibition, we will wish to note that Helson distinguishes adaptation level theory not only from neuronal fatigue, but also from the biological concepts of homeostasis and equilibrium. Helson contended that homeostatic processes (such as those which regulate body temperature) are distinct from adaptation level theory insofar as they tend toward a constant and fixed inner state, whereas perceptual adaptation is labile and takes on a new 'zero' value with reference to a continua of stimuli. The goal of adaptation level, according to Helson, is not restful equilibrium, but the creation of a reference point for the evaluation of other stimuli. Note that in this sense adaptation level is distinct from 'error-correction' as well, for it assumes no fixed point of 'truth.' This important idea that adaptation creates a flexible reference point for purposes of information processing is also central to Barlow's model, as is the importance of intracortical inhibition.

Barlow and Foldiak (1989) proposed that the visual coding of information was kept segregated by inhibition between different kinds of information. For example, in the case of the McCollough effect, inhibitory connections might 
connect both red and green to vertical. If red verticals are constantly presented to the visual system, then the inhibitory connections between them are stipulated to gain strength. As a result, red is inhibited in the presence of any vertical grating, and this produces a sensation of the opponent color, green, as in the McCollough Effect. Of course, red and green have inhibitory connections to horizontal, and other inhibitory links exist between these and other visual dimensions. A schematic illustration of the model is shown in Fig. 18.

Figure 18. Schematic illustration of Barlow's (1990) model of contingent adaptation. based on inter-channel inhibition. Barlow's model accomplishes the functional goals of recalibration using the modification of inhibitory connections. At top left, the system is shown in its initial, balanced state. Correlated adaptation input is represented at top right by the non-orthogonality of the two relevant dimensions. As a result of contingent adaptation, the inhibitory connections are modified, as shown in the lower center. After pairing red with dense texture, for example, and green with sparse, the mutually inhibitory connections between these values increase, increasing the apparent orthogonality of features, but actually unbalancing the system and producing a contingent aftereffect. This scheme may help produce a more efficient visual code (Barlow and Foldiak, 1989;

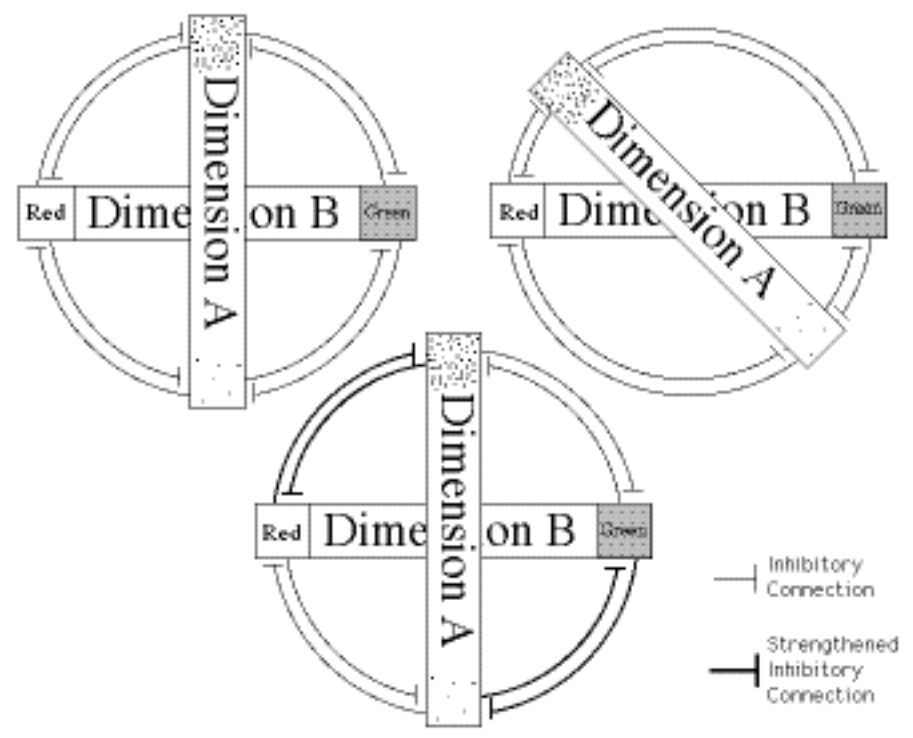
Foldiak, 1990).

It should be noted that Barlow (1990) explicitly suggests that his theory is not intended to contradict either associative theories of contingent adaptation (broadly construed), nor theories that attribute specific functionality to the McCollough Effect. For example, edge-contingent color adaptation may help to eliminate retinal color fringes produced by chromatic aberrations in the lenses of the eyes. As Barlow puts it, 'The argument presented here does not contradict these suggestions, but I shall approach the problem from a different angle' (1990, p. 368). What Barlow is advocating is a theory of adaptation to the joint activation of sets of features (cf. also MacKay and MacKay, 1974), and the model he proposes involves the modification of inhibitory connections.

A further observation to be made is that although Barlow's (1990) theory regarding the McCollough Effect is not an error-correction model, it bears a striking resemblance in many details to the one proposed by Dodwell and Humphrey (1990). Specifically, Dodwell and Humphrey regard their model as an adaptation-level theory (Helson, 1964) applied to the correlations between features. Barlow's model is described by him as an adaptation to joint occurrences, and thus has the same effective theoretical notion embedded in it. Although Barlow's model is not presented as an error-correction model, but rather as a multi-dimensional normalization function, it may accomplish much of what a calibrationist would desire (Note 8).

The real distinction between the Dodwell and Humphrey (1990) formulation and Barlow's (1990) is probably that Dodwell and Humphrey regard error-correction (featural decorrelation) as a goal in itself (cf. also Anstis, 1975), whereas the mutual inhibition model entails two other specific goals having to do with information processing. Barlow has suggested that the normal relationships between self-motion, stereopsis, motion parallax, and size, for example, are determined by the 3-D world, such that 'normal' perception (and this is where perceptual errorcorrection seems to be accomplished by the model) should find the co-occurrence of appropriate concomitant changes in correlated variables invisible. But this process has two further goals (or, less teleologically, 'fortunate outcomes'). One of these goals, presented by Barlow and Foldiak (1989) is to produce sparse, orthogonal visual dimensions to make the coding of sensory information efficient. The second, described most clearly by Barlow (1990), is the goal of making novel relationships or correlations in the environment stand out as distinct from what is 
normal.

Barlow (1990) likens the effect of this sort of system to the automatic gain control of the inhibitory connections that allow the visual system to code relative luminance (contrast) rather than absolute luminance. Such automatic gain control is postulated to allow the visual system to make much finer discriminations (by representing deviations from the current norm) than it could possibly make if it attempted to represent the whole range of visible luminances at once. Similarly, by adapting to relationships between features, the visual system can accomplish normalization of inter-feature correlation structure which then allows it to represent deviations from this multi-dimensional relational norm. It is clear that this scheme allows for great gains in overall sensitivity to departures from representational structures.

To review, what we have found most compelling about Barlow's (1990) model of contingent adaptation is that it seems to have all of the characteristics one could desire:

- it is simple, elegant, and very close to being neurophysiologically plausible (Note 9);

- it accomplishes the goals of perceptual calibration automatically;

- it maximizes information representation.

This last feature of the model is perhaps the most important and is achieved in two ways. (1) In the first place, the model decorrelates the various visual dimensions automatically and keeps them orthogonal to one another. By working with orthogonal features, one can represent more information. (This is true because if two features were correlated then knowing the value of both would not provide as many bits of information as knowing the value of two orthogonal dimensions.) (2) The second gain for information processing is that the visual system, thus construed, becomes adapted to the prevailing correlations and cancels them out. As a result it becomes most sensitive to novel contingencies in the environment as departures from the norm.

Although it is possible to trace to Helson (1964) the idea that adaptation levels represent a platform from which other stimuli can be efficiently assessed, only in more modern proposals such as Dodwell and Humphrey's (1990) and Barlow's (1990; Barlow and Foldiak, 1989) has this idea been applied to interactions between visual dimensions. Moreover, Barlow's model is more explicit both about a possible mechanism and about the informational gain it can achieve. Barlow and Foldiak's proposal combines into a single conceptual model a demonstration of how the modifiable inhibition between 'channels' of visual information can produce an efficient system both for detecting and representing correlational structure in the environment and for thereby representing novelty in the environment as deviations from a correlational norm (cf. also Kohonen and Oja, 1976).

\section{Conclusions from the contingent adaptation of texture density}

Because of our presently limited understanding of how texture density might be coded in vision -- whether by some non-linear signal of texture (e.g. texton) energy or by a non-Fourier measure of spatial scale (Durgin, 1995b) -theories of the contingent density aftereffects are unsatisfactorily constrained. It makes quite a difference whether density is conceived as a metric of other features' strength or as a feature in itself. For example, in texton theory (e.g. Julesz, 1981), texton density is the measure of texture intensity. Under this conception, the adjustment of perceived density is a sort of textural gain control which, apparently, is distinct from gain control applied to the contrast energy of individual texture elements (Durgin, 1995a; Durgin and Hammer, 1994, 1995). However, a different conception can be adopted. After all, the output of the texture density 'system' is interpreted perceptually as 
a spatial measure of texture separation. From this view-point it is spatial scale that has ultimately been shown to be affected by both simple adaptation and adaptation contingent on (1) color information presented in an adjacent region, (2) order of texture presentation, and (3) texture element orientation. In this section we will examine these two views of density (feature energy and spatial scale) in turn and see how consideration of each might facilitate a coherent description of visual learning and adaptation generally.

What kinds of visual learning will we consider? We have outlined three kinds of visual learning: Plasticity, as illustrated by the work of Karni and Sagi $(1991,1993)$ was shown to be essential for improved performance in the discrimination of forms defined by texture boundaries between differently oriented elements. Error-correction (Andrews, 1964) or recalibration can be illustrated by various pieces of evidence for structural change or synaptic modification having to do with the perception of alignment (Poggio et al., 1992) and local orientation in visual space (Wolfe and O'Connell, 1986). Finally, we have discussed contingent adaptation (e.g. McCollough, 1965) and suggested that the best current theories of this include ideas of recalibration (Dodwell and Humphrey, 1990; Held, 1980) but also of interchannel inhibition (Barlow, 1990; Barlow and Foldiak, 1989).

We have reviewed or presented three different contingent adaptation paradigms in which the local perceived density of textures was made contingent on some factor seemingly extraneous to density itself. Although we think the relation of these effects to ideas of error-correction is evident, we will try to show in the remainder of this section that other kinds of learning, such as improved texture discrimination, might be explained by similar principles. Let us now consider the issues from each of the two views of texture density.

\section{Density as a measure of texture}

In texton and energy models of texture perception the basis of texture discrimination is differential texton density or filter energy. We have seen that filter energy and density are dissociable in the sequence-contingent effect (Durgin and Hammer, 1994, 1995; cf., also Durgin, 1995a), and it would probably be fairly straightforward to introduce a nonlinearity into a filter theory to produce this distinction. For example, the total activity of some pool of detectors might be divided by the local peak activity to produce a contrast-independent metric of density. Note that some such move is already implicit in texton theory, which keeps texture contrast and texture density coded separately. In this view, adaptation of a specific texture feature, such as vertical bars should not intrinsically change its apparent density: Although resulting threshold elevation might reduce the total energy from the texture, the peak energy would be reduced proportionally, as well. Thus, density and energy models of texture perception are probably quite distinct entities.

A texture-specific change in texture density, such as demonstrated in Section 2, changes the texture value of the specific texture type which has been trained. Recall that Karni and Sagi (1991) demonstrated an improvement in texture boundary discrimination that was specific to the background texture used. It is evident that such an effect could be explained by a shift in the texture value (i.e. represented density) of that background texture type. Because their learning procedure involved repeated exposure to dense oriented textures which are primarily made up of the 'background' texture, one interpretation of visual learning in texture discrimination could be based entirely on a element-specific modification of the textural value of the background texture elements. Note that although we have not demonstrated longevity of contingent density effects, simple density aftereffects do seem to produce long term changes and Wolfe and O'Connell (1986) demonstrated a long term tilt aftereffect which could be related to orientation-specific texture adaptation.

If the Helson/Barlow adaptation idea is applied directly to the Karni and Sagi situation, a further observation can be made: Given a continual barrage of a particular type of texture, the representation of that texture becomes redundant and therefore superfluous. Foldiak (1990) has shown how an inhibitory learning network will reduce the number of input units used to code the most frequent members of the input stimulus set. In Barlow's terms, the common stimulus will become invisible, and deviations from the norm will become more distinct. Regarded in this way, Karni and Sagi's demonstration of long term learning in preattentive texture segregation may be ultimately 
prove to be an example of the kind of increased perceptual sensitivity that Barlow and Foldiak's model can accomplish.

The same theory that accounts for orientation-specific density aftereffects can also handle color-specific density aftereffects described by Durgin (in press). Although the color information was presented in a spatially segregated region to avoid confounding it with achromatic texture information, even a small amount of retinal color spreading (Poppel, 1986) would be sufficient to produce color-biasing of the texture analyzers at the boundaries of the textured regions, for example.

We began this subsection with a presumption that density might be a measure of texture energy. It is worth stressing that the way that argument has been interpreted here, the reduction of perceived texture density is not due to a failure to detect individual elements, for example (i.e. threshold elevation). Instead, we are proposing only that density itself be treated as an input to a texture discrimination process (a lá Julesz) and that a change in the perceptual effect of the same input due to a local change in adaptation level can be interpreted as a kind of automatic gain control. Thus, the interpretation we are giving is one that involves treating density as a feature in itself. The gain control is not applied in order to increase sensitivity to changes in density itself, however (as with luminance adaptation), but rather, as Barlow argues, to increase sensitivity to deviations from a complex correlational structure of features over time. Our suggestion for interpreting Karni and Sagi's (1991) texture boundary learning effect involves treating the background texture as being 'adapted out' for the texture system, so that the novel (target) texture pops out more readily from the surround.

\section{Density as spatial scale}

A second manner of viewing texture density is to consider it a measure of non-Fourier size or spatial scale (cf., Durgin, 1995b). On this view the simple adaptation effects derive from efforts to normalize the spatial scale of textures across the visual field. If correlations between spatial scale and other features are present, then perceived spatial scale will come to be modified by the presence of those other features as well.

The local recalibration of spatial scale is, in principle, similar to the calibration of alignment and orientation evidenced in visual learning in hyperacuity tasks (Poggio et al., 1992). Although it is beyond the scope of this article to analyze the computational models that can accomplish such spatial learning, it is probably worth mentioning that the method employed by Poggio et al. employed feedback (i.e. the learning was supervised). One of the goals of Barlow and Foldiak's formulation is to remove external 'supervision' from learning. It is imaginable that some form of feedback would come to be available about size scaling across the retina or local alignment just from the movement of the eyes, but it is clear in any case that methods of perceptual adaptation and error correction can be implemented without explicit supervision, and that these methods are quite efficient (Foldiak, 1990) at coding the information necessary to represent regularities in the environment (such as straight lines, and regions of homogenous spatial scale).

For experiments in which density was made contingent on either texture element orientation or surrounding color, it is possible that associations between some correlate of spatial scale and the cueing features did in fact grow mutually inhibitory. In general, it can be seen that describing density as spatial scale does not alter the general account of adaptation to correlation that we have been describing. The argument for a spatial scale interpretation appears vulnerable to the following criticism, however: In all of the prior cases we have discussed, the adaptations appear to occur in visual area V1. We are unfamiliar with any evidence that there is a representation of non-Fourier size in primary visual cortex. It may be that the visual information that is affected by texture adaptation is used for the later representation of visual scale. On this view, texture density would be latently represented early on. On the other hand, the normal course of perception may involve the synthesis of spatial scale information that is extracted at different stages of visual processing.

\section{Implications for theories of texture perception}


The evidence presented in the second section of this article have demonstrated that texture perception is modifiable in a variety of surprising ways. Although roughly consistent with (non-linear) filter theories, the results also support texton-like theories of texture perception in that density appears to be coded quite independently of texture energy. On the other hand, this is not to say that any particular set of textons are going to turn out to be the 'right' ones. Adaptive flexibility may be the rule.

Field's $(1993,1994)$ theories of texture perception include the notion that the visual system must use a sparse code to represent textures if it is to succeed efficiently, and that the code the visual system uses is ideally suited to represent the images it is faced with. A similar thesis regarding the importance of sparse representation, though less developed with respect to natural images, has been advanced by Foldiak (1990). Foldiak showed that a network model with inhibitory connections structured as in the model of Barlow and Foldiak (1989) would create a sparse code for representing sets of images (he used lines in one simulation and alphanumeric symbols in another). In conjunction with the modifiability of texture perception that has emerged throughout our investigations, these ideas seem to us to strongly implicate the importance of mechanisms, such as that proposed by Barlow and Foldiak, which can adapt to correlations and thereby produce a sparse encoding mechanism in the form of orthogonal visual feature analyzers.

It is quite conceivable that the visual processing of texture is adaptive in the complex manner suggested by Barlow's model. Under this hypothesis, contingent adaptation represents both (1) an adaptation to correlational structures and (2) a scheme for orthogonalizing visual texture analyzers. Such a scheme would appear to be of great value in generating a sparse representational code (Foldiak, 1990).

\section{General Conclusions}

We have reported several facets of a fairly new aftereffect and used it to provide new evidence of the visual system's compensatory response to correlations between visual features. Though the kinds of computational learning mechanisms that exist in the human brain may have an astonishing variety, Helson's principle of adaptation level and Barlow's model of adaptation offer a good deal of promise for understanding these responses as an important type of visual learning.

In support of Barlow's (1990) contention that these sorts of mechanisms make associative learning possible, we have also shown that intermodal correlations seem to be interpreted as informative about the world rather than as a basis for error-correction. Though much of our discussion has been limited to a speculative treatment of the nature of the modifications discovered, we hope we have made a plausible case for the importance of this kind of learning in visual perception, and suggested that, rather than an annoying error of the visual machinery with which we are endowed, adaptation effects may demonstrate a most remarkable functional property of our visual apparatus.

Vision works to efficiently and effectively encode information. The learning it does is often to increase sensitivity where sensitivity is most needed. The sensitivity it gains is not only to particular features, but also to conjunctions among features -- or rather deviations from those conjunctions. This is not done by mapping the inputs into a preordained set of features, but rather by fitting the visual code to the input. The contingent aftereffects of texture described here may be evidence of the visual system's effort to retune its coding scheme. The adaptability of texture density that we have demonstrated suggests that density information may play a very important role in the visual analysis of texture.

\section{References}

Ahissar, M. and Hochstein, S. (1993). Attentional control of early perceptual learning. Proc. Nat. Acad. Sci. USA 90, 
5718-5722.

Allan, L. G. (1984). Contingent aftereffects in duration judgements. In Timing and time perception. J. Gibbon and L. Allan (Eds.). Ann. New York Acad. Sci. 423, 116-130.

Allan, L. G. and Siegel, S. (1986). McCollough effects as conditioned responses: Reply to Skowbo. Psychol. Bull.100, 388-393.

Allan, L. G. and Siegel, S. (1993). McCollough effects as conditioned responses: Reply to Dodwell and Humphrey. Psychol. Rev. 100, 342-346.

Allan, L. G., Siegel, S., Collins, J. C. and MacQueen, G. M. (1989). Color aftereffect contingent on text. Percept. Psychophys. 46, 105-113.

Andrews, D. P. (1964). Error-correcting perceptual mechanisms. Q. J. Exp. Psychol. 16, 104-115.

Anstis, S. M. (1967). Visual adaptation to gradual change in intensity. Science 155, 710-712.

Anstis, S. M. (1974). Size adaptation to visual texture and print: Evidence for spatial-frequency analysis. Am. J. Psychol. 87, 261-267.

Anstis, S. M. (1975). What does visual perception tell us about visual coding? In Handbook of Psychobiology. M. S. Gazzaniga and C. Blakemore (Eds.). Academic Press, New York, pp. 269-323.

Anstis, S. M. and Harris, J. P. (1974). Movement aftereffects contingent on binocular disparity. Perception 3, 153168.

Ballard, D. and De Sa, V. (1994). Learning classification through correlated multi-modality experience. Proceedings of the 1994 CVS Symposium, Rochester, NY, p. 18.

Barlow, H. B. (1990). A theory about the functional role and synaptic mechanism of visual after-effects. In Vision: Coding and Efficiency. C. Blakemore (Ed.). Cambridge University Press, Cambridge, England, pp. 363-375.

Barlow, H. B. and Foldiak, P. (1989). Adaptation and decorrelation in the cortex. In The computing neuron. R. M. Durbin, C. Miall and G. J. Mitchison (Eds.). Addison-Wesley, Wokingham. pp. 54-72.

Barlow, H. B. and Hill, R. M. (1963). Evidence for a physiological explanation of the waterfall phenomenon and figural after-effects. Nature 200, 1345-1347.

Bedford, F. (1993). Perceptual Learning. In The psychology of learning and motivation, Vol. 30. D. Medin (Ed.). Academic Press, San Diego, CA, pp. 1-60.

Bedford, F. (1995). Constraints on perceptual learning: Objects and dimensions. Cognition 54, 253-297.

Bedford, F. and Reinke, K. S. (1993). The McCollough effect: Dissociating retinal from spatial coordinates. Percept. Psychophys. 54, 515-626.

Bergen, J. and Adelson, E. (1988). Visual texture segmentation based on energy measures. J. Opt.Soc.Am. A3, 98101.

Blakemore, C. and Campbell, F. W. (1969). On the existence of neurons in the human visual system selectively sensitive to the orientation and size of retinal images. J. of Physiol. 203, 237-260.

Blakemore, C., Carpenter, R. and Georgeson, M. (1970). Lateral inhibition between orientation detectors in the human visual system. Nature 228, 37-39. 
Blakemore, C., Carpenter, R. and Georgeson, M. (1971). Lateral thinking about lateral inhibition. Nature 234, 418419.

Broerse, J. and Grimbeek, P. (1994). Eye movements and the associative basis of contingent color aftereffects: A comment on Siegel, Allan, and Eissenberg (1991). J. Exp. Psychol.: Gen. 123, 81-85.

Broerse, J. and O'Shea, R. P. (1995). Local and global factors in spatially-contingent coloured aftereffects. Vision Res. 35, 207-226.

Broerse, J., Shaw, C., Dodwell, P. and Muir, D. (1994). Colored aftereffects contingent upon global transformations? Spat. Vis. 8, 95-117.

Burgess, A. and Barlow, H. B. (1983). The precision of numerosity discrimination in arrays of random dots. Vision Res. 23, 811-820.

Carlson, C. R., Moeller, J. R. and Anderson, C. H. (1984). Visual illusions without low spatial frequencies. Vision Res. 24, 1407-1413.

Chubb, C., Sperling, G. and Solomon, J. A. (1989). Texture interactions determine perceived contrast. Proc.Nat.Acad. Sci. USA 86, 9631-9635.

Cornelissen, F. W. and Kooijman, A. C. (1994). Interactions between modulated luminance patterns and random dot patterns. Vision Res. 34, 2561-2568.

de Sa, V. (1994). Unsupervised classification learning from cross-modal environmental structure. Unpublished doctoral dissertation, University of Rochester, Rochester, NY.

De Valois, K. K. and Switkes, E. (1980). Spatial frequency specific interactions of dot patterns and grating. Proc.Nat.Acad. Sci. USA 77, 662-665.

Dodwell, P. C. (1994). On raising our sights. Spat. Vis. 8, 9-17.

Dodwell, P. C. and Humphrey, G. K. (1990). A functional theory of the McCollough effect. Psychol. Rev. 97, 78-89.

Dodwell, P. C. and Humphrey, G. K. (1993). What is important about McCollough effects? Reply to Allan and Siegel. Psychol. Rev. 100, 347-350.

Dodwell, P. C. and O'Shea, R. P. (1987). Global factors generate the McCollough effect. Vision Res. 27, 569-580.

Durgin, F. H. (1992). Interocular transfer of texture density aftereffects. Invest. Ophthalmol. Vis. Sci.33, 959.

Durgin, F. H. (1995a). Interocular transfer of aftereffects of texture density and texture contrast. (Manuscript in preparation).

Durgin, F. H. (1995b). Texture density adaptation and the perceived numerosity and distribution of texture. J. Exp. Psychol.: Human Percept. Performance 21, 149-169.

Durgin, F. H. (in press) Visual aftereffect of texture density contingent on color of frame. Percept. Psychophys.

Durgin, F. H. and Hammer, J. T., (1994). Density aftereffect contingent on temporal sequence. Invest. Ophthalmol. Vis. Sci. 35, 1838.

Durgin, F. H. and Hammer, J. T., (1995). Visual aftereffects of sequential perception: Texture density and texture brightness. (Manuscript in preparation). 
Durgin, F. H. and Harp, C. J. (1995). Perceptual learning and the rapid auditory cueing of visual attention: Evidence for a criterion shift. (Manuscript submitted for publication).

Durgin, F. H. and Proffitt, D. R. (November, 1991). Detection of texture density: Evidence from adaptation. Paper presented at the annual meeting of the Psychonomic Society, San Francisco.

Ellis, S. R. (1976). Orientation constancy of the McCollough effect. Percept. Psychophys. 19, 183-192.

Emerson, V. F., Humphrey, G. K. and Dodwell, P. C. (1985). Colored aftereffects contingent on patterns generated by the Lie transformation groups. Percept. Psychophys. 37, 155-162.

Favreau, O. E., Emerson, V. and Corballis, M. (1972). Motion perception: A color-contingent aftereffect. Science 176, 78-79.

Field, D. J. (1993). Scale-invariance and self-similar 'wavelet' transforms: An analysis of natural scenes and mammalian visual systems. In Wavelets, fractals and Fourier transforms: New developments and applications. Farge, M., Hunt, J. and Vassilicos (Eds.). Oxford University Press, Oxford, .

Field, D. J. (1994). What is the goal of sensory coding? Neural Computation 6, 559-601.

Foldiak, P. (1990). Forming sparse representations by local anti-Hebbian learning. Biol. Cybernet. 64, 165-170.

Garcia-Perez, M. A. (1991). Visual phenomena without low spatial frequencies: A closer look. Vision Res. 31, 16471653.

Gibson, J. J. (1933). Adaptation, after-effect, and contrast in the perception of curved lines. J. Exp. Psychol. 16, 131.

Gibson, J. J. (1966). The senses considered as perceptual systems. Lawrence Erlbaum Associates, Hillsdale, NJ.

Gilden, D. L., Bertenthal, B. I. and Othman, S. (1990). Image statistics and the perception of apparent motion. J. Exp. Psychol.: Human Percept. Performance 16, 693-705.

Graham, N. V. S. (1989). Visual pattern analyzers. Oxford University Press, New York.

Harp, C. J., Lewis, D. A. and Durgin, F. H. (1995). Can auditory stimuli become exogenous cues for covert visual orienting? Invest. Ophthalmol.Vis. Sci., 36, S372.

Harris, C. S. (1970). Effect of viewing distance on a color aftereffect specific to spatial frequency. Psychonom. Sci. 21, 350. (Abstract)

Harris, C. S. (1980). Perceptual plasticity in the human adult. In Visual Coding and Adaptability. C. S. Harris (Ed.). Lawrence Earlbaum Associates, Hillsdale, NJ, pp. 95-149.

Held, R. (1980). The rediscovery of adaptability in the visual system: Effects of extrinsic and intrinsic chromatic dispersion. In Visual Coding and Adaptability. C. S. Harris (Ed.). Lawrence Earlbaum Associates, Hillsdale, NJ, pp. 69-94.

Held, R. and Shattuck, S. (1971). Color- and edge-sensitive channels in the human visual system: Tuning for orientation. Science 174, 314-316.

Helson, H. (1964). Adaptation-level theory: An experimental and systematic approach to behavior. Harper \& Row, New York.

Hepler, N. (1968). Color: A motion-contingent aftereffect. Science 162, 376-377. 
Houck, M. R. and Hoffman, J. E. (1986). Conjunction of color and form without attention: Evidence from an orientation-contingent color aftereffect. J. Exp. Psychol.: Human Percept. Performance 12, 186-199.

Humphrey, G. K. (in press) The McCollough effect: Misperception and reality. In Visual constancies: Why things look as they do. V. Walsh and J. Kulikowski (Eds.). Cambridge University Press, Cambridge, England.

Humphrey, G. K., Dodwell, P. C. and Emerson, V. F. (1985). The roles of orthogonality and color contrast in the generation of pattern-contingent color aftereffects. Percept. Psychophys. 38, 343-353.

Humphrey, G. K., Dodwell, P. C. and Emerson, V. F. (1989). Pattern-contingent color aftereffects on non-induced patterns. Percept. Psychophys. 45, 97-109.

Humphrey, G. K., Goodale, M. A. and Gurnsey, R. (1991). Orientation discrimination in a visual form agnosic: Evidence from the McCollough effect. Psychol. Sci. 2, 331-335.

Humphrey, G. K., Gurnsey, R. and Fekete, E. (1991). Rapid discrimination of McCollough effects. Perception 20, 467-482.

Humphrey, G. K., Gurnsey, R. and Bryden, P. J. (1994). An examination of colour-contingent pattern aftereffects. Spat. Vis. 8, 77-94.

Humphrey, G. K., Herbert, A. M., Symons, L. A. and Kara, S. (1994). McCollough effect to 'Form': A local phenomenon. J. Exp. Psychol.: Gen. 123, 86-90.

Humphrey, G. K., Skowbo, D. Symons, L. A., Herbert, A. M. and Grant, C. L. (1994). Text-contingent coloraftereffects: A re-examination. Percept. Psychophys. 56, 405-413.

Humphrey, G.K., Goodale, M. A., Corbetta, M. and Aglioti, S. (1995). The McCollough effect reveals orientation discrimination in a case of cortical blindness. Curr.Biol. 5, 545-551.

Jones, P. D. and Holding, D. H. (1975). Extremely long-term persistence of the McCollough effect. J. Exp. Psychol.: Human Percept. Performance 1, 323-327.

Julesz, B. (1981). Textons, the elements of texture perception, and their interactions. Nature, 290, 89-97.

Karni, A. and Sagi, D. (1991). Where practice makes perfect in texture discrimination: Evidence for primary visual cortex plasticity. Proc. Nat. Acad. Sci. USA 88, 4966-4970.

Karni, A. and Sagi, D. (1993). The time course of learning a visual skill. Nature 365, 250-252.

Kohonen, T. and Oja, E. (1976). Fast adaptive formation of orthogonalizing filters and associative memory in recurrent networks of neuron-like elements. Biol. Cybernet. 21, 85-95.

Lovegrove, W. J. and Over, R. (1972). Color adaptation of spatial frequency detectors in the human visual system. Science 176, 541-543.

MacKay, D. M. (1964). Central adaptation in mechanisms of form vision. Nature 203, 993-994.

MacKay, D. M. and MacKay, V. (1974). The time course of the McCollough Effect and its physiological implications. J. of Physiol. 237, 38-39.

MacKay, D. M. and MacKay, V. (1975). What causes decay of pattern-contingent chromatic aftereffects? Vision Res. $15,462-464$.

Malik, J. and Perona, P. (1990). Preattentive texture discrimination with early visual mechanisms. J. Opt. Soc. Am. 
A7, 923-932.

Marr, D. (1982). Vision: A computational investigation into the human representation and processing of visual information. Freeman, New York.

Mayhew, J. E. W. (1973). Aftereffects of movement contingent on direction of gaze. Vision Res. 13, 1789-1795.

Mayhew, J. E. W. and Anstis, S. M. (1972). Movement aftereffects contingent on color, intensity, and pattern. Percept. Psychophys. 12, 77-85.

McCarter, A. and Silver, A. (1977). The McCollough effect: A classical conditioning phenomenon? Vision Res. 17, 317-319.

McCollough, C. (1965). Color adaptation of edge-detectors in the human visual system. Science 149, 1115-1116.

McCollough, C. (1994). Do McCollough effects provide evidence of global pattern processing? Invest. Ophthalmol. Vis. Sci.35, 1489.

McGurk, H. and MacDonald, J. (1976). Hearing lips and seeing voices. Nature 264, 746-748.

McKee, S. P. and Westheimer, G. (1978). Improvement in vernier acuity with practice. Percept. Psychophys. 24, 258-262.

Michael, C. R. (1978). Color vision mechanisms in monkey striate cortex: Simple cells with dual opponent-color receptive fields. J. of Neurophysiol. 41, 1233-1241.

Montalvo, F. S. (1976). A neural network model of the McCollough effect. Biol. Cybernet. 25, 49-56.

Mulligan, J. B. and MacLeod, D. I. A. (1988). Reciprocity between luminance and dot density in the perception of brightness. Vision Res. 28, 503-519.

Murch, G. M. (1972). Binocular relationships in a size and color orientation specific aftereffect. J. Exp. Psychol. 93, 30-34.

Murch, G. M. (1976). Classical conditioning of the McCollough effect: Temporal parameters. Vision Res. 16, 615619.

Over, R., Broerse, J, Crassini, B. and Lovegrove, W. (1974). Orientation-specific aftereffects and illusions in the perception of brightness. Percept. Psychophys. 15, 53-56

Pelli, D. G. and Zhang, L. (1991) Accurate control of contrast on microcomputer displays. Vision Res. 31, 13371350.

Poggio, T., Fahle, M. and Edelman, S. (1992). Fast perceptual learning in visual hyperacuity. Science 256, 10181021.

Poppel, E. (1986). Long-range colour-generating interactions across the retina. Nature 320, 523-525.

Potts, M. J. and Harris, J. P. (1975). Movement aftereffects contingent on the pattern or color of a stationary surround. Vision Res. 15, 1225-1230.

Ramachandran, V. A. and Braddick, O. (1973). Orientation-specific learning in stereopsis. Perception 2, 371-376.

Regan D. and Beverly, K. I. (1978). Illusory motion in depth: Aftereffect of adaptation to changing size. Vision Res. 18, 209-212. 
Rescorla, R. A. and Wagner, A. R. (1972). A theory of Pavlovian conditioning: Variations in the effectiveness of reinforcement and nonreinforcement. In Classical conditioning II. A. H. Black and W. F. Prokasy (Eds.). AppletonCentury-Crofts, New York, pp. 64-99.

Sagi, D. (June, 1994). Spatial interactions and plasticity in early vision. Paper presented at the 19th CVS Symposium, Rochester, NY.

Savoy, R. L. (1984). 'Extinction' of the McCollough effect does not transfer interocularly. Percept. Psychophys. 36, 571-576.

Schoups, A., Orban, G. A., Vogels, R, Tootell, R., Schilz, C., Dubois, S., and Roucoux, A. (1995). Neuronal changes underlying orientation discrimination learning. Perception 24, supplement, 21.

Sharpe, L. T., Harris, J. P., Fach, C. C. and Braun, D. I. (1991). Contingent aftereffects: Lateral interaction between color and motion. Percept. Psychophys. 49, 434-447.

Siegel, S. and Allan, L. G. (1985). Overshadowing and blocking of the orientation-contingent color aftereffects: Evidence for a conditioning mechanism. Learning Motiv. 16, 125-138.

Siegel, S. and Allan, L. G. (1992). Pairings in learning and perception: Pavlovian conditioning and contingent aftereffects. Psychol. Learning Motiv. 28, 127-160.

Siegel, S., Allan, L. G. and Eissenberg, T. (1992). The associative basis of contingent color aftereffects. J. Exp. Psychol.: Gen. 121, 79-94.

Siegel, S., Allan, L. G. and Eissenberg, T. (1994). Scanning and form-contingent color aftereffects. J. Exp. Psychol.: Gen. 123, 91-94.

Skowbo, D. (1984). Are McCollough effects conditioned responses? Psychol. Bull. 96, 215-226.

Skowbo, D. (1986). McCollough Effects as conditioned responses? Reply to Allan and Siegel. Psychol. Bull. 100, 394-397.

Skowbo, D., Gentry, T., Timney, B. and Morant, R. B. (1974). The McCollough effect: Influence of several kinds of visual stimulation on decay rate. Percept. Psychophys. 16, 47-49.

Sloane, M. E., Ost, J. W., Etheridge, D. B. and Henderlite, S. E. (1989). Overprediction and blocking in the McCollough effect. Percept. Psychophys. 45, 110-120.

Stromeyer, C. F. III. (1978). Form-color after-effects in human vision. In Handbook of sensory physiology , Vol 8. R. Held, H. W. Leibowicz and H. L. Teuber (Eds.). Springer, New York, pp. 97-142.

Thompson, P. and Latchford, G. (1986). Colour-contingent after-effects are really wavelength-contingent. Nature $320,525-526$.

Thompson, P. G. and Movshon, J. A. (1978). Storage of spatially specific threshold elevation. Perception 7, 65-73.

Thompson, P. and Travis, D. (1989). Making Mayhew and Frisby effortlessly discriminable. Perception 18, $231-235$.

Tolhurst, D. (1972). Adaptation to square-wave gratings: Inhibition between spatial frequency channels in the human visual system. J. of Physiol. 226, 231-248.

Vautin, R. G. and Berkley, M. A. (1977). Responses of single cells in cat visual cortex to prolonged stimulus movement: Neural correlates of visual aftereffects. J. of Neurophysiol. 40, 1051-1065. 
Walker, J. (1966). Textural aftereffects: Tactual and visual. Unpublished doctoral dissertation, University of Colorado, Boulder.

Walker, J. (1972). A texture-contingent visual motion aftereffect. Psychonom. Sci. 28, 533-535.

Walker, J. T. and Irion, A. L. (1979). Two new contingent aftereffects: Perceived auditory duration contingent on pitch and on temporal order. Percept. Psychophys. 26, 241-244.

Walker, J. T., Irion, A. L. and Gordon, D. G. (1981). Simple and contingent aftereffects of perceived duration in vision and audition. Percept. Psychophys. 29, 475-486.

Westbrook, R. F. and Harrison, W. (1984). Associative blocking of the McCollough effect. Q. J. Exp. Psychol. 103, 668-674.

White, K. D., Petry, H., Riggs, L. A. and Miller, J. (1978). Binocular interactions during establishment of McCollough effects. Vision Res. 18, 1201-1215.

Wolfe, J. M. (1990). See as thou wast wont to see: Aftereffects and the recalibration of visual perception. Paper presented at the 17th CVS Symposium, Rochester, NY.

Wolfe, J. M. and O'Connell, K. M. (1986). Fatigue and structural change: Two consequences of visual pattern adaptation. Invest. Ophthalmol. Vis. Sci.27, 538-543.

Yasuda, K. (1978). Color aftereffects contingent on MacKay complementary regular patterns. Jap. Psychol. Res. 20, 115-123.

\section{Acknowledgments}

This work was supported by a faculty research grant from Swarthmore College. Some of the findings have been presented previously at Annual Meetings of the Psychonomic Society, in San Francisco, 1991 and in Washington, DC, 1993. Portions of this article are based on a doctoral dissertation completed at the University of Virginia by Frank H. Durgin. We are indebted to G. K. Humphrey, M. Ahissar, T. J. Olson and B. I. Berthenthal for many useful comments and suggestions along the way, to S. A. Jacquot, who helped with programming the displays, and to J. T. Hammer who helped to conduct several of the experiments.

\section{Footnotes}

1. Experiment 1 was reported at the annual meeting of the Psychonomic Society in San Francisco, 1991. (back to text)

2. The luminance profile shown in Fig. 3 for a square balanced dot is idealized because on a typical raster monitor, the luminance of a pixel is determined, in part, by the luminance of the pixel to its immediate left. The dots are therefore not perfectly symmetrical in their luminance profile, as depicted, nor are the exact pixel luminances known. To call a dot balanced, we require only that the addition or subtraction of many hundreds of dots not change the average luminance output of the monitor as measured photometrically. Although such dots are not perfectly structured, they do have the advantage of being truly luminance balanced, whereas band-pass filtering stimuli on such a monitor would produce systematic errors if the non-independence of adjacent pixel values were not 
adequately controlled for. (back to text)

3. Some of Denis Pelli's 'VideoToolBox' routines were used in for the presentation of stimuli, (cf., Pelli and Zhang, 1991). (back to text)

4. Stuart Anstis has described to FD an unsuccessful attempt to produce aftereffects to changing spatial frequency, June, 1994. (back to text)

5. While it is true that Karni and Sagi's (1991) effects do not show interocular transfer (but see Schoups et al., 1995), their testing conditions do not entail dichoptic presentations intended to eliminate rivalry. Durgin (1992) found that the interocular transfer of texture density aftereffects could be reduced significantly by patching the unadapted eye during adaptation and the adapted eye during testing. (back to text)

6. Because a raster monitor writes horizontally across the screen, the actual luminance of any pixel is affected by both its own defined color (gray level) and that of its neighbor to the left (See Note 2). The effect of this can be dramatically illustrated by comparing the luminance of a one-pixel-wide white vertical line amidst black, to a onepixel-wide white horizontal line amidst black. The horizontal line will be roughly twice as luminous. For this reason, separate adjustments (gray scale values for the annuli) had to be made for horizontal and vertical textures. Although the precise distribution of luminance about each texture element on a standard monitor is not easy to control, the processing of luminance-balancing a dense texture does accomplish the goal of removing much of the low spatialfrequency information. (back to text)

7. E.g. Shepard Siegel, in a presentation at the annual meeting of the Psychonomic Society in Washington DC, 1993, suggested that any stimulus, including auditory stimuli, could probably be used to produce contingent color aftereffects. There is no evidence to date of such an effect. (back to text)

8. For example, Barlow suggests that typically concomitant sensations such as the change in the vergence of the eyes and change in retinal size with the approach of an object may be jointly normalized by a scheme of the type he proposes. Thus he argues that size constancy (the scaling of perceived size to compensate for viewing distance) can be produced automatically from interactions between changing-size and changing-vergence information. As a result, perceived size will be modulated by vergence in much the same way that McCollough effect demonstrates the modulation of color by orientation. (back to text)

9. The model depends on the strengthening of inhibitory connections between simultaneously activated units. Such effects have not been generally observed, though they may eventually be documented. It should be possible to substitute an intermediate excitatory connection (that drives the inhibitory one). (back to text)

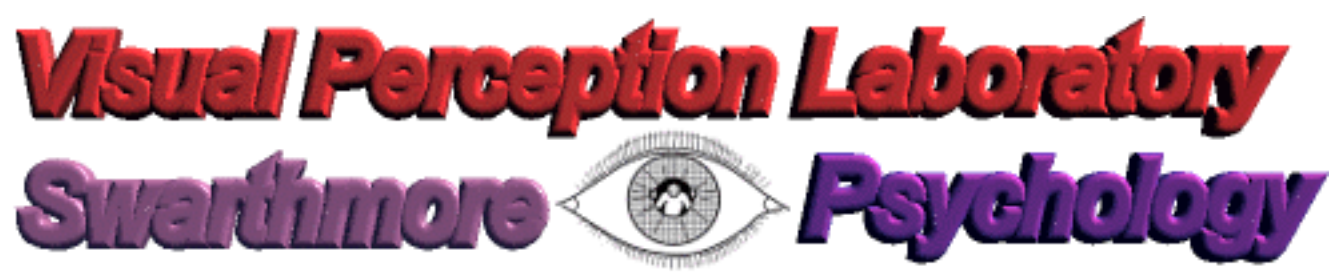

Created by Richa Jain, rjain1@swarthmore.edu 